\title{
ANALYZING STARBURSTS USING MAGELLANIC CLOUD STAR CLUSTERS AS SIMPLE STELLAR POPULATIONS
}

\author{
ANDREW J. LEONARDI ${ }^{1}$ \\ Department of Physics and Astronomy, CB 3255, University of North Carolina, Chapel Hill, NC 27599; \\ and Division of Natural Sciences and Engineering, University of South Carolina Spartanburg, Spartanburg, SC 29303; \\ aleonardi@gw.uscs.edu \\ AND \\ JAMES A. Rose \\ Department of Physics and Astronomy, CB 3255, University of North Carolina, Chapel Hill, NC 27599; \\ jim@physics.unc.edu \\ Received 2002 September 10; accepted 2003 June 19
}

\begin{abstract}
Integrated spectra have been obtained of 31 star clusters in the Magellanic Clouds (MC) and of four Galactic globular clusters. The spectra cover the wavelength range 3500-4700 $\AA$ at a resolution of $3.2 \AA$ FWHM. The MC clusters primarily cover the age range from less than $10^{8}$ to about $3 \mathrm{Gyr}$ and hence are wellsuited to an empirical study of aging poststarburst stellar populations. An age-dating method is presented that relies on two spectral absorption feature indices, $\mathrm{H} \delta / \mathrm{Fe}$ I $\lambda 4045$ and $\mathrm{Ca}$ II, as well as an index measuring the strength of the Balmer discontinuity. We compare the behavior of the spectral indices in the observed integrated spectra of the MC clusters with that of indices generated from theoretical evolutionary synthesis models of varying age and metal abundance. The synthesis models are based on those of Worthey, when coupled with the combination of an empirical library of stellar spectra by Jones for the cooler stars and synthetic spectra, generated from Kurucz model atmospheres, for the hotter stars. Overall, we find good agreement between the ages of the MC clusters derived from our integrated spectra (and the evolutionary synthesis modelling of the spectral indices) and ages derived from analyses of the cluster color-magnitude diagrams, as found in the literature. Hence, the principal conclusion of this study is that ages of young stellar populations can be reliably measured from modelling of their integrated spectra.

Key words: galaxies: starburst — galaxies: star clusters — Magellanic Clouds
\end{abstract}

\section{INTRODUCTION}

While it is convenient to assume that most galaxies have experienced a simple star formation history, for example, exponentially declining over time, there is growing evidence that many galaxies have experienced multiple major episodes of concentrated star formation (hereafter, starbursts) during their lifetimes, as opposed to a smoothly varying star formation rate. Since the seminal study of Searle, Sargent, \& Bagnuolo (1973), in which starbursts were advocated to explain the extreme $U B V$ colors of the bluest galaxies, starbursts have been implicated in a variety of situations in which galaxies have been found with blue colors and/or enhanced Balmer absorption-line strengths. Briefly, Dressler \& Gunn (1983) proposed starbursts to explain the photometrically blue galaxies discovered by Butcher \& Oemler (1978a, 1978b) in galaxy clusters at $z \sim 0.3$, starbursts have been advocated to explain the excess of blue galaxies at faint apparent magnitudes (e.g., Ellis 1997), and both numerical simulations of interacting and merging galaxies (Toomre \& Toomre 1972; Mihos \& Hernquist 1994, 1996) and observations of such systems (e.g., Larson \& Tinsley 1978; Kennicutt et al. 1987; Rifatto et al. 2001 and references therein) indicate that starbursts should be common in merger events. Clearly, there is much to be gained from obtaining information on the ages of the starbursts in these situations.

\footnotetext{
${ }^{1}$ Present address: Division of Natural Sciences and Engineering, University of South Carolina Spartanburg, Spartanburg, SC 29303.
}

Unfortunately, reliably extracting the ages of starburst episodes using population synthesis modelling has proven to be problematic for several reasons. First, there is the difficulty in uniquely detecting the young stellar population (YSP) produced in a starburst against the backdrop of an underlying old stellar population (OSP). This problem manifests itself as a degeneracy in the age versus the strength of a burst. Specifically, a younger, weaker burst is difficult to distinguish from an older, stronger burst (e.g., Couch \& Sharples 1987; Bica, Alloin, \& Schmidt 1990; Charlot \& Silk 1994; Leonardi \& Rose 1996). The second problem is the degeneracy between age and metal abundance. While this degeneracy is most notorious in the case of modelling older stellar populations (Worthey 1994), it afflicts younger populations as well (Nolan, Dunlop, \& Jimenez 2001). Adding to these two difficulties is the confusing effect of interstellar reddening on the spectral energy distribution (SED) of a burst. Finally, there is the complicating factor that the behavior of the SED and absorption-line strengths of a poststarburst population is so nonlinearly dependent on age, with rapid evolution back to near normal colors taking place over the first few gigayears followed by a much more gradual evolution thereafter (Searle, Sargent, \& Bagnuolo 1973; Bica et al. 1990; Charlot \& Silk 1994). In short, age dating of starburst populations is confronted by serious difficulties.

Despite the above-mentioned challenges a variety of studies have been conducted to extract the ages of starburst populations from population synthesis modelling (Bruzual 1983; Bica et al. 1990; Schweizer \& Seitzer 1992; Bernlöhr 
1992; Leonardi \& Rose 1996; Kurth, Fritze-v. Alvensleben, \& Fricke 1999; Bicker, Fritze-v. Alvensleben, \& Fricke 2002). These investigations range in emphasis from broadband photometry to relatively high-resolution studies of specific spectral features.

The ideal testing ground for models of the integrated spectra of starburst populations is the observed integrated spectra of star clusters and, in particular, of star clusters in the Magellanic Clouds (MCs). Clusters are in essence ministarbursts, given that the stars in a cluster are coeval and of uniform chemical composition. They are particularly advantageous if their stellar density is high enough to eliminate confusion from the underlying OSP in the galaxy. Clusters in the Clouds serve the purpose well, spanning a wide enough range in age and metallicity (e.g., Sagar \& Pandey 1989) to adequately assess the behavior of stellar population indicators. They are particularly useful because there is no corresponding set of clusters in the Galaxy with sufficient diversity in both age and metallicity. Milky Way globular clusters, while adequately encompassing metallicity space, are all old systems. Stellar populations of appropriate age are found in Galactic open clusters, but they are not dense and populous enough to be readily observed in integrated light above the confusion of stars in the Galactic disk. In contrast, the young, populous clusters in the Clouds represent excellent analogs of a starburst YSP. MC clusters are distant and populous enough to be studied in integrated light but are still sufficiently nearby so that the results of integrated light techniques can be compared with the fundamental age and metallicity information obtained from color-magnitude diagrams (e.g., Kerber et al. 2002) and from spectroscopy of individual cluster members (e.g., Olszewski et al. 1991), including high-dispersion (Hill et al. 2000; Spite et al. 2001).

Given the attractiveness of MC clusters, there have been surprisingly few studies of their integrated spectra. The rationale behind early integrated light studies was to calibrate cluster age under the photometric classification scheme of Searle, Wilkinson, \& Bagnuolo 1980; hereafter SWB). In their landmark paper SWB used two reddeningindependent parameters created from integrated Gunn photometry to show that MC clusters fall along a onedimensional sequence in this parameter space. SWB divided the sequence into seven photometric classification groups, which, because of their usefulness, are still referred to today. A comparison with a similar sequence for Galactic clusters led SWB to propose that the sequence is due to the combined effects of age and chemical composition. Soon thereafter Rabin (1982) demonstrated the usefulness of combining measurements of the equivalent widths of both Balmer and metal-line absorption features, along with population synthesis modelling of these features, to assign ages to the SWB classes. In addition, Cohen, Rich, \& Persson (1984) used the International Ultraviolet Explorer (IUE) to study the integrated spectra of $17 \mathrm{MC}$ clusters in the ultraviolet. More recently, age and metallicity determinations based on spectroscopic indices defined by the Lick group (Faber et al. 1985) have been derived for a sample of 14 MC clusters (de Freitas Pacheco, Barbuy, \& Idiart 1998). However, the most comprehensive studies to date of the behavior of MC clusters from the near-infrared to the nearultraviolet have been carried out by Bica, Alloin, and collaborators (Bica \& Alloin 1986a, 1986b; Bica, Alloin, \& Santos 1990; Bica et al. 1990). The purpose of their studies was to use the integrated spectra of MC clusters as direct templates for galaxy evolution models. Very recently Beasley, Hoyle, \& Sharples (2002) have obtained optical integrated spectra for $24 \mathrm{MC}$ clusters with the FLAIR multifiber spectrograph on the UK Schmidt telescope, covering a large range in cluster ages and metallicities.

The goal of this study is to develop and test integrated light indicators, which accurately detect and characterize the age and metallicity of a YSP. Specifically, we characterize the behavior of diagnostic spectral absorption-line indices generated from evolutionary synthesis models employing a combined empirical and synthetic spectral stellar library. The age and metallicity dependence of the spectral indicators in the synthesis models are then compared with observed indices in the integrated spectra of a sample of MC clusters. This paper represents an extension of the use of spectral indicators originally modeled in Leonardi \& Rose (1996, hereafter LR96) and further refined in Leonardi \& Worthey (2000). In LR96 we demonstrated how the degeneracy between age and burst strength can be lifted, assuming solar composition populations, in age-dating poststarburst galaxies in the Coma cluster of galaxies. In Leonardi \& Worthey (2000) a preliminary approach was made in regard to the age-metallicity degeneracy in detecting a young, metal-rich population in the candidate merger galaxy NGC 5018. In this paper we present a detailed analysis of the effects of age versus metallicity on the integrated indices of starburst models, and we compare them with the observed indices of MC clusters of known age and $[\mathrm{Fe} / \mathrm{H}]$. In $\S 2$ we address the MC cluster selection criteria and our sources for their ages and $[\mathrm{Fe} / \mathrm{H}]$. In $\S 3$ we summarize the spectral indicators used in this study. The details of the empirical and synthetic spectral libraries are given in $\S 4$, while the evolutionary synthesis models are described in $\S 5$. In $\S 6$ we discuss the determination of ages and metallicities of MC clusters from our integrated spectra and models, and in $\S 7$ we briefly consider our results in regard to the history of cluster formation in the LMC.

\section{THE MC CLUSTER SAMPLE}

When LR96 investigated the degeneracy between young, weak starbursts and old, strong starbursts, which exists when typical optical indicators are analyzed (Couch \& Sharples 1987; Schweizer \& Seitzer 1992), the population synthesis models employed were restricted to solar metallicity. A limited metallicity analysis indicated that a difference of 0.4 dex in metallicity can result in a $40 \%$ difference in derived age for ages around $1 \mathrm{Gyr}$, even if the strength of the burst is held constant. Hence, the chemical composition of the YSP must be known before an accurate age can be determined. To test diagnostic indices that can distinguish age from metallicity effects, a grid of star clusters with a wide range in both age and metallicity is required. The young, populous MC clusters span a significant range in metallicity within the effective age boundaries (0-3 Gyr) established in LR96.

\subsection{Selection Criteria}

The indices used in LR96 and described in $\S 3$ were demonstrated to be effective for YSPs younger than $\sim 2-3$ Gyr (LR96). Targets were initially selected from Sagar \& Pandey (1989), which supplies a compilation of ages and metallicities derived from color-magnitude diagrams in the 
literature. Each candidate cluster was then viewed by eye on film copies of the blue plate ESO/SERC Southern Sky Atlas and evaluated for contamination from stars belonging either to the MC field or to the Galactic foreground. Compact populous clusters were primarily selected since they present a true portrayal of an integrated light population; i.e., observations will suffer minimally from stochastic difficulties due to a few dominant stars and/or background subtraction problems.

The final sample consists of 28 LMC clusters and three SMC clusters. Added to the sample for comparison purposes are four Galactic globular clusters.

\subsection{Observations}

All clusters were observed at the Cerro Tololo InterAmerican Observatory (CTIO) over five nights in 1995 November. The data were obtained with a Cassegrain spectrograph attached to the $1.5 \mathrm{~m}$ telescope at CTIO. The detector was a Loral $1200 \times 800$ pixel CCD camera with a pixel size of $15 \mu \mathrm{m}$ used with the 400 line $\mathrm{mm}^{-1}$ grating No. 58 in second order. This combination provides a reciprocal dispersion of $1.12 \AA$ pixel $^{-1}$ and an average spectral resolution of $3.2 \AA$ (FWHM). The effective spectral range is $\sim 3420-4760 \AA$. A slit of size $460^{\prime \prime} \times 3^{\prime \prime}$, with a fixed east-west orientation, was used for all the clusters.

Typical exposure times were 45 minutes for each cluster. Dome flats and HeAr lamps were observed for flat-field correction and wavelength calibration. Spectrophotometric standards LTT 377 and EG 21 were measured through a wide slit at the beginning and end of each night for flux calibration. Positions for the LMC clusters were taken from Olszewski et al. (1988) and Kontizas et al. (1990). SMC cluster positions are from Welch (1991).

To obtain a true integrated light measurement, the slit was manually trailed across each cluster in the north-south direction during the exposure between specified limits. The $\log$ of the observations, including the amount that the slit was trailed, is given in Table 1.

Image reductions were carried out using the $\mathrm{IRAF}^{2}$ package in the standard manner: bias subtraction, flat-field correction, spectra extraction, wavelength and flux calibration. All cluster spectra are shown in Figure 1.

\subsection{Adopted Ages and Metallicities}

The point of this paper is to test whether ages and metallicities derived directly from evolutionary synthesis models of the integrated spectral indices of MC clusters are in accord with those determined from a "fundamental" analysis, i.e., one based on cluster color-magnitude diagrams (CMDs) and on individual star spectroscopy. Thus, to carry out such a comparison, we require a set of reliably known ages and metallicities for the MC clusters. To minimize the possible sources of discrepancies, we desire the literature values that we adopt to be as homogeneous as possible. Though there has been a long history of analyzing MC clusters, few large studies of multiple clusters have been undertaken. Both observational and theoretical difficulties in the techniques have given rise to disparate estimates of both the ages and metallicities, and so the field suffers from

\footnotetext{
${ }^{2}$ IRAF is distributed by the National Optical Astronomy Observatory, which is operated by the Association of Universities for Research in Astronomy, Inc., under cooperative agreement with the National Science Foundation.
}

a lack of consistency. Attempts to compile the various measurements in single bibliographies (e.g., Sagar \& Pandey 1989; Seggewiss \& Richtler 1989) have proved useful, but compilations in and of themselves do not solve the discrepancies. Therefore, the selection of literature sources must be undertaken carefully. The rationale for the adopted ages and metallicities of the MC cluster sample is described below.

\subsubsection{Cluster Ages}

Ideally, an age comparison set would consist of a large sample of MC cluster ages derived from primary age indicators, which are defined here as indicators developed from the analysis of star clusters in color-magnitude diagrams (CMDs). However, the time-consuming effort needed to assemble a CMD, as well as the difficulty for ground-based photometry to reach the $V \sim 23 \mathrm{mag}$ level needed to detect the main-sequence (MS) turnoff in the older MC clusters, has prohibited any single study from including more than a few clusters. Instead, a major effort has gone into assembling compilations of ages based on MS turnoffs (Hodge 1981, 1982; Sagar \& Pandey 1989). Though they are useful as a starting point, the lack of uniformity in MS turnoff ages necessitates their careful use as a comparison set. Since homogeneity is the fundamentally desired characteristic of our comparison set, we instead turn to secondary age indicators based on the integrated properties of the clusters, which have been calibrated against a primary age indicator. In particular, a secondary age indicator is desired that is easily measurable over a large sample of clusters, is robust to stochastic sampling effects, and is applicable over a wide range of ages. Optical colors satisfy all these criteria, and the available data for MC clusters is extensive. Frenk \& Fall (1982) showed that a parallel for the SWB diagram exists in $U B V$ photometry. It is equivalent to the SWB sequence with the same class divisions when $U-B$ color is plotted versus $B-V$ color. Elson \& Fall (1985) parameterized the sequence by drawing a smooth curve through the clusters, dividing the curve into equal increments, and assigning a value to each cluster by projecting it onto the curve: their $s$ parameter. The final calibration of $s$ with age is based solely on ages derived from MS turnoff determinations (Elson \& Fall 1988). Subsequently, the $s$ parameter was refined further (Girardi et al. 1995; Girardi \& Bertelli 1998) by more precisely assigning the $s$ value to a given cluster, accounting for color dispersion effects cause by stochastic fluctuations in younger clusters and metallicity differences in older clusters. The modifications improved the treatment of older clusters, as well as outliers in the color-color diagram. With the great increase in reliable integrated $U B V$ photometry for LMC clusters (Bica, Clariá, \& Dottori 1992; Bica et al. 1996), the set of ages based on the $s$ parameter has become the largest and most homogeneous sample of LMC cluster ages in the literature. We choose this set to be the basis of age comparison for this paper. Ages of the clusters are taken preferentially from Girardi et al. (1995) and Girardi \& Bertelli (1998) and then from Elson \& Fall (1988). Adopted ages are listed in Table 2.

\subsubsection{Cluster Metallicities}

To this date, studies measuring homogeneous metallicities for a large number of MC clusters have been sparse. In fact, the only one with a significant sample of clusters is that 
TABLE 1

OBSERVATION LOG

\begin{tabular}{|c|c|c|c|c|c|}
\hline Cluster & R.A. (J2000.0) & Decl. (J2000.0) & Obs. Date & $\begin{array}{l}\text { Exp. Time } \\
\text { (s) }\end{array}$ & $\begin{array}{l}\text { Trail Width } \\
\quad(\operatorname{arcsec})\end{array}$ \\
\hline \multicolumn{6}{|c|}{ SMC } \\
\hline NGC $411 \ldots$. & 10614.70 & -720058.40 & 1995 Nov 24 & 2700 & 40 \\
\hline NGC 416 .................... & 10751.00 & -722129.50 & 1995 Nov 23 & 2700 & 20 \\
\hline NGC 419 .................. & 10809.90 & -725322.20 & 1995 Nov 22 & 2700 & 60 \\
\hline \multicolumn{6}{|c|}{ LMC } \\
\hline NGC $1651 \ldots \ldots \ldots \ldots . . . .$. & 43748.00 & -704034.60 & 1995 Nov 24 & 3600 & 50 \\
\hline NGC $1751 \ldots$ & 45427.80 & -695242.20 & 1995 Nov 22 & 2700 & 40 \\
\hline NGC $1754 \ldots \ldots \ldots \ldots . . .$. & 45442.10 & -703105.60 & 1995 Nov 20 & 1800 & 20 \\
\hline NGC $1777 \ldots$ & 45654.90 & -742128.30 & 1995 Nov 22 & 2700 & 30 \\
\hline NGC $1783 \ldots$ & 45856.90 & -660340.10 & 1995 Nov 22 & 2100 & 50 \\
\hline NGC 17 .............. & 50001.10 & -695223.30 & 1995 Nov 24 & 3600 & 40 \\
\hline NGC $1806 \ldots \ldots \ldots \ldots$ & 50214.40 & -680333.10 & 1995 Nov 22 & 2700 & 50 \\
\hline NGC $1818 \ldots \ldots \ldots \ldots . . . . .$. & 50404.40 & -662936.50 & 1995 Nov 23 & 2700 & 30 \\
\hline NGC $1831 \ldots \ldots \ldots \ldots . . . .$. & 50559.80 & -645923.60 & 1995 Nov 20 & 2700 & 53 \\
\hline NGC $1846 \ldots \ldots \ldots \ldots . . . .$. & 50733.40 & -673038.10 & 1995 Nov 21 & 2700 & 90 \\
\hline NGC $1866 \ldots \ldots \ldots \ldots . . . .$. & 51324.30 & -653135.50 & 1995 Nov 23 & 1200 & 50 \\
\hline NGC $1868 \ldots \ldots \ldots \ldots . . .$. & 51412.70 & -640032.00 & 1995 Nov 21 & 2700 & 24 \\
\hline NGC 1978............... & 52838.30 & -661547.90 & 1995 Nov 22 & 2700 & 50 \\
\hline NGC $2010 \ldots \ldots \ldots \ldots . . . .$. & 53103.30 & -705047.20 & 1995 Nov 24 & 2700 & 60 \\
\hline NGC $2133 \ldots \ldots \ldots \ldots . . .$. & 55205.50 & -711045.60 & 1995 Nov 20 & 2700 & 30 \\
\hline 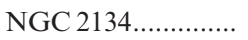 & 55232.50 & -710603.90 & 1995 Nov 21 & 2700 & 40 \\
\hline NGC $2136 \ldots$. & 55316.70 & -692937.70 & 1995 Nov 23 & 2700 & 30 \\
\hline NGC $2155 \ldots \ldots \ldots \ldots \ldots$ & 55820.10 & -652839.40 & 1995 Nov 22 & 2700 & 30 \\
\hline NGC $2162 .$. & 60008.90 & -634309.70 & 1995 Nov 22 & 2700 & 30 \\
\hline NGC $2164 \ldots \ldots \ldots \ldots \ldots$ & 55906.80 & -683115.30 & 1995 Nov 23 & 2700 & 30 \\
\hline NGC $2173 \ldots \ldots \ldots \ldots \ldots$ & 55856.60 & -725823.90 & 1995 Nov 20 & 2700 & 42 \\
\hline 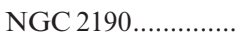 & 60224.00 & -744256.20 & 1995 Nov 21 & 2700 & 46 \\
\hline 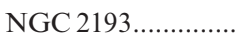 & 60603.30 & -650518.30 & 1995 Nov 22 & 2700 & 20 \\
\hline NGC 2203................. & 60612.80 & -752520.00 & 1995 Nov 24 & 2700 & 60 \\
\hline 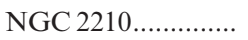 & 61146.90 & -690614.20 & 1995 Nov 23 & 2700 & 30 \\
\hline NGC 2213................ & 61121.90 & -713047.70 & 1995 Nov 20 & 2700 & 23 \\
\hline NGC $2214 \ldots \ldots \ldots \ldots . . . .$. & 61305.40 & -681444.20 & 1995 Nov 23 & 2700 & 24 \\
\hline NGC $2249 \ldots \ldots \ldots \ldots$ & 62600.80 & -685322.70 & 1995 Nov 23 & 2700 & 30 \\
\hline \multicolumn{6}{|c|}{ Galactic Globulars } \\
\hline 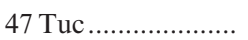 & 02357.90 & -720436.60 & 23 Nov 23 & 600 & 50 \\
\hline M15.. & 213001.30 & 121039.10 & 20 Nov 20 & 1200 & 60 \\
\hline M79. & 52208.40 & -243428.40 & 21 Nov 21 & 1200 & 40 \\
\hline 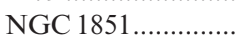 & 51230.00 & -400532.30 & 20 Nov 20 & 900 & 50 \\
\hline
\end{tabular}

Note.- Units of right ascension are hours, minutes, and seconds, and units of declination are degrees, arcminutes, and arcseconds.

of Olszewski et al. (1991). In that work, equivalent widths of the infrared calcium triplet $(\lambda \sim 8500 \AA)$ of one or two stars per cluster are used to derive metallicities for $\sim 80$ clusters. In this paper Olszewski et al. (1991) is the adopted reference for MC cluster metallicities whenever possible. For other clusters metallicities are taken from the two major compilations of MC cluster data (Sagar \& Pandey 1989; Seggewiss \& Richtler 1989), as noted.

\section{DIAGNOSTIC SPECTRAL INDICES}

\subsection{Strategy for Defining Spectral Indices}

It is now widely acknowledged that the cleanest way to decouple age from metallicity effects in the integrated spectra of stellar populations is to combine the measurement of a Balmer line strength, which responds primarily to the temperature (hence, age) of the MS turnoff, with a metal line strength, which is primarily sensitive to the temperature (hence, metallicity) of the giant branch (e.g., Rabin 1982; Worthey 1994; Buzzoni, Mantegazza, \& Gariboldi 1994). In addition, there is an advantage to using the higher order Balmer lines $(\mathrm{H} \gamma, \mathrm{H} \delta$, etc. $)$ in order to maximize the contribution of the MS turnoff stars to the integrated light (relative to the contribution of the GB) and also to minimize any contamination from emission lines. However, obtaining clean measurements of the strengths of these spectral features is challenging, as a result of line crowding, especially in the vicinity of the higher order Balmer lines. Here we briefly consider different strategies for measuring spectral features before describing the indices to be used in this study.

A particular difficulty for defining spectral indices in the case of poststarburst populations is the great range in 


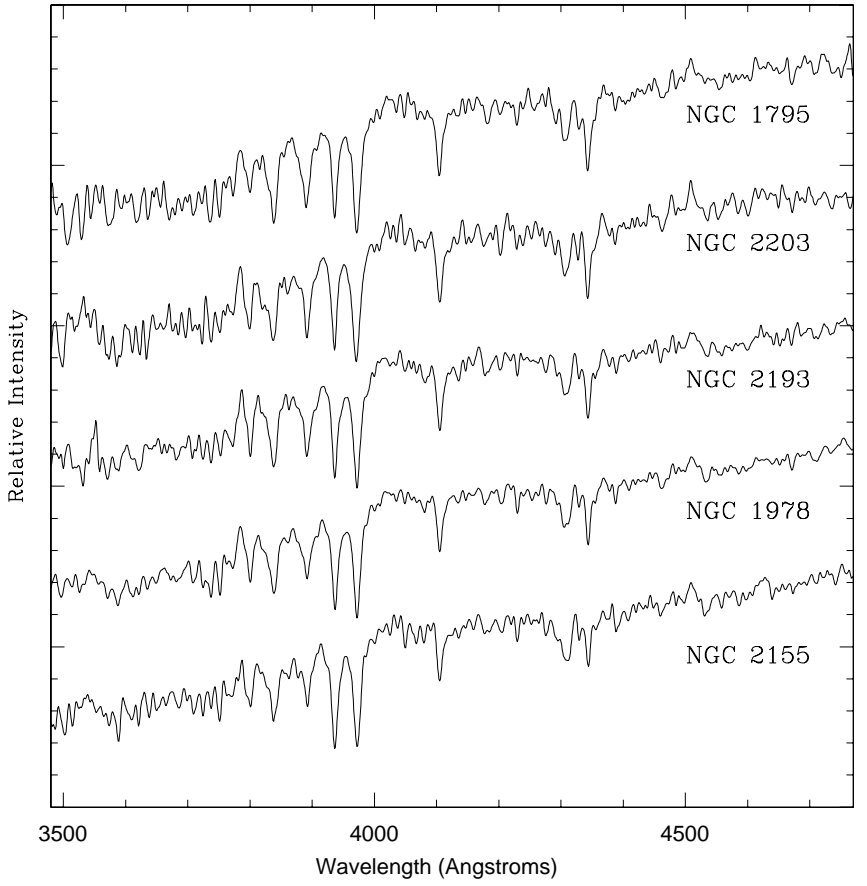

FIG. $1 a$

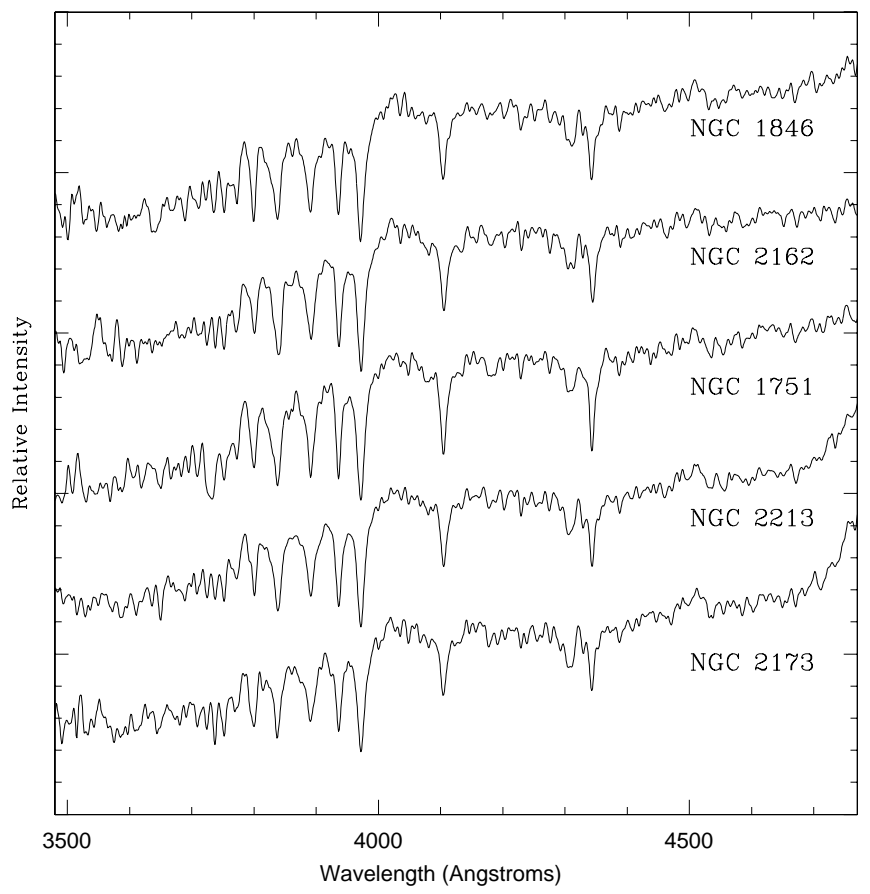

FIG. $1 c$

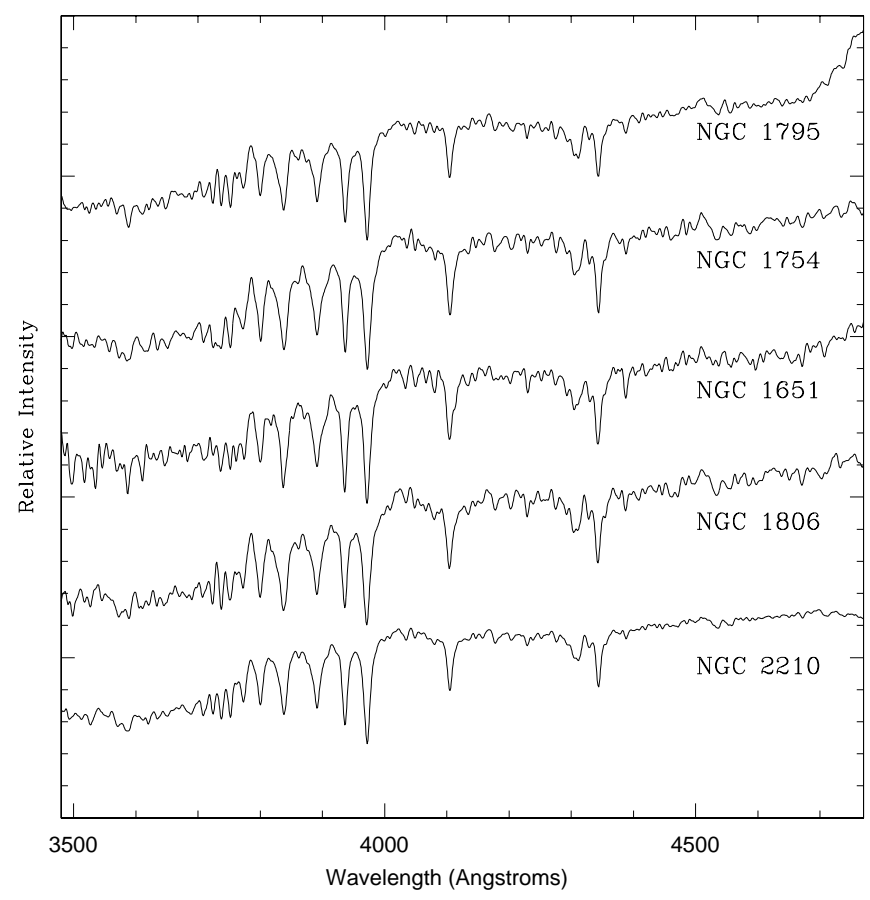

FIG. $1 b$

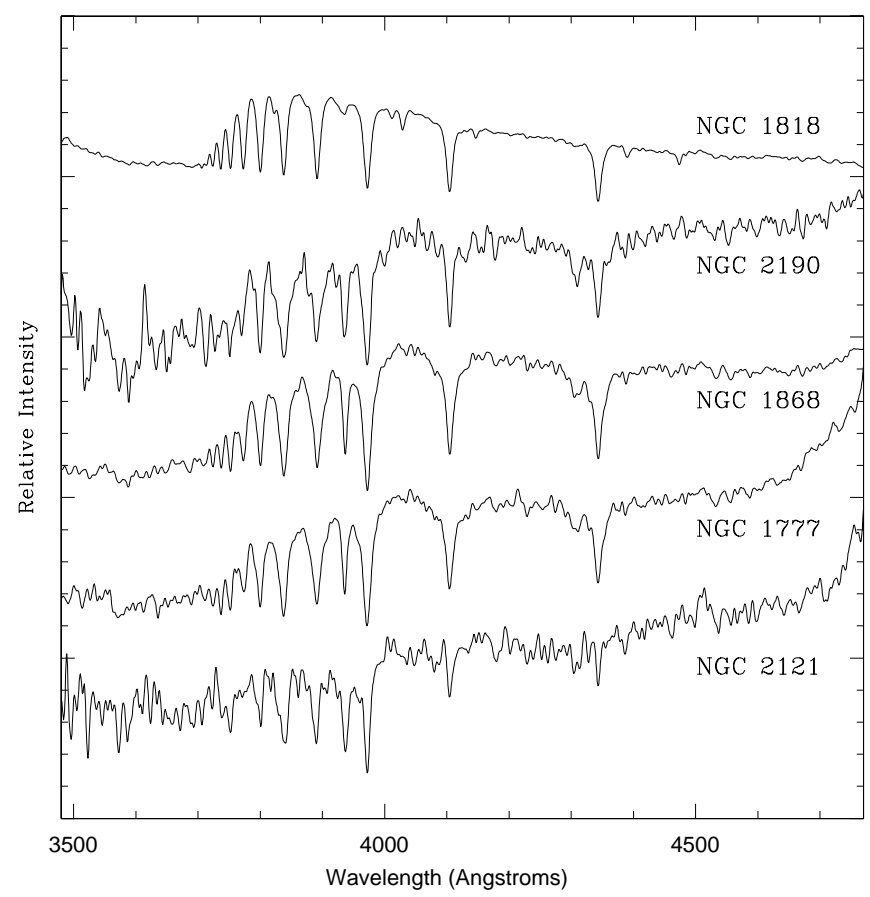

FIG. 1d

FIG. 1.-Observed MC cluster spectra smoothed with a Gaussian of width $2 \AA$. The spectra have been put in the order of increasing Ca II index (see $\S 3.1$ ) from the bottom to the top of each panel. $(a)-(f)$ LMC clusters. $(g)$ SMC clusters. $(h)$ Galactic globular clusters.

spectral types that they cover. The problem is that one is potentially looking at the superposition of two fundamentally different spectra, a young population, as illustrated by NGC 1777 in Figure $1 d$, and an old population, as illustrated by 47 Tuc in Figure 1 $h$. Naturally, it is important to use spectral indicators that can track the behavior of the desired features over this great range in spectral type. Examination of NGC 1777 reveals that to measure the total equivalent width (EW) of a Balmer line in this spectrum requires using continuum sidebands that are widely separated from the line center because of the extensive Balmer line wings. In contrast, in the spectrum of 47 Tuc the Balmer line wings are lost amid the proliferation of metal lines that dominate this cooler composite population. An additional problem is that, where the Balmer lines dominate in the young populations, the metal lines are very weak. Hence, where the Balmer lines best lend themselves to definition through wide-bandpass EW indicators, the metal lines are 


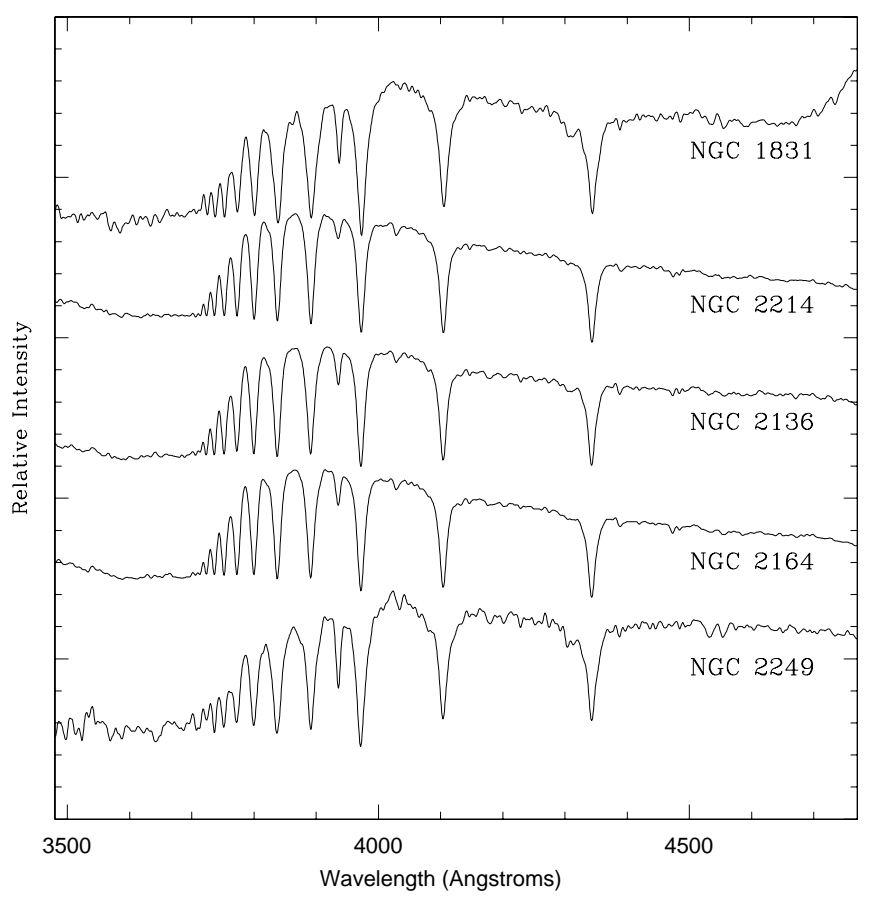

FIG. 1e

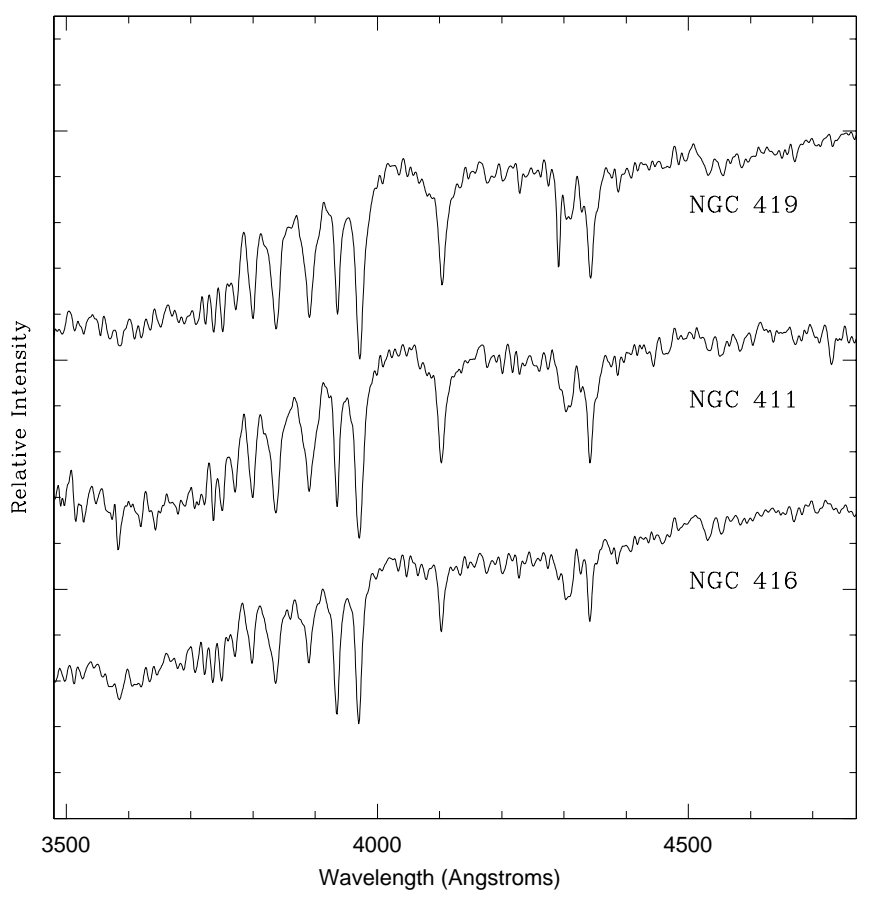

FIG. $1 g$

so weak as to be difficult to characterize in that manner. In addition, defining the appropriate sidebands in the case of the $\mathrm{Ca}$ II $\mathrm{H}$ and $\mathrm{K}$ lines, which we will see are highly useful as age/metallicity indicators, is difficult as a result of the steep gradient in the pseudocontinuum across these features.

In optimizing the definition of indices it is important to keep in mind that an EW index will be independent of spectral resolution (an important advantage) if the sidebands are located out beyond the line wings and the line bandpass includes the entire line wings. If, however, this criterion is

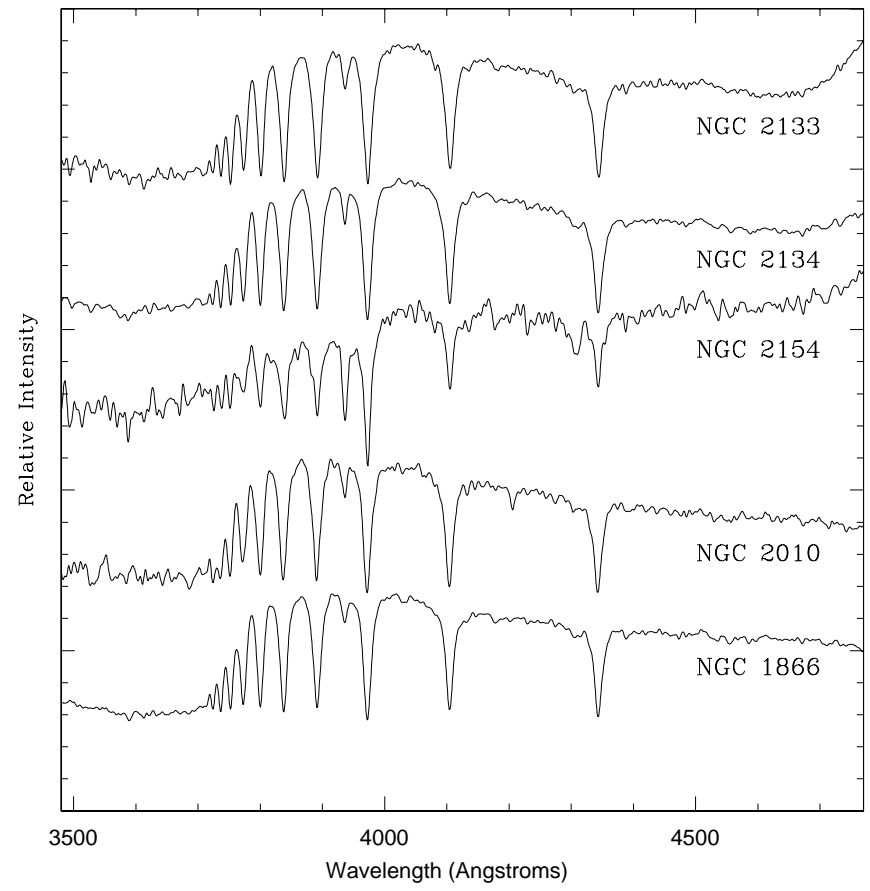

FIG. $1 f$

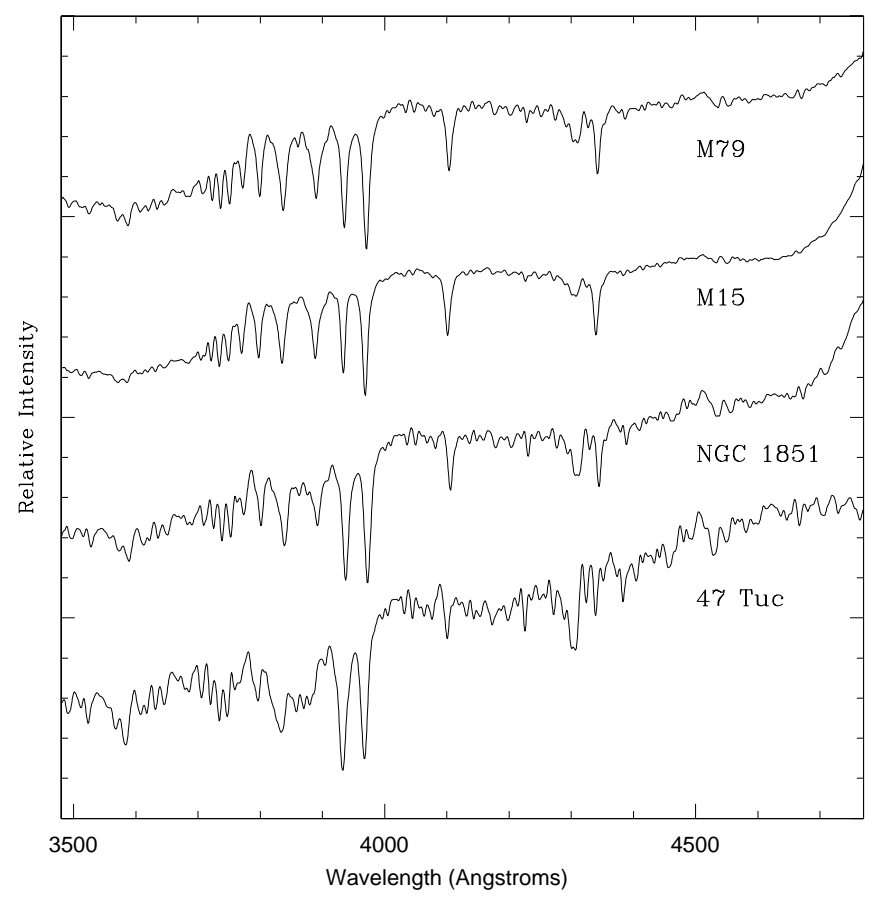

FIG. $1 h$

not met, then the EW index can be highly sensitive to line broadening (either instrumental or Doppler), as the flux deficit in the line is redistributed out of the line bandpass and into the sidebands with increasing broadening. Thus, the dilemma is that, for the older populations, one would like to narrow the line definition as much as possible, to isolate the contribution of the particular feature from the increasingly confusing contribution of other features, a problem that afflicts not only the line bandpass but the sidebands as well. However, this strategy has the drawback of making the EW very sensitive to spectral resolution and in addition leads to 
TABLE 2

MC Cluster Adopted Ages and Metallicities

\begin{tabular}{|c|c|c|c|c|c|}
\hline Cluster & $\begin{array}{l}\text { SWB } \\
\text { Type }^{\mathrm{a}}\end{array}$ & $\begin{array}{l}\text { Age } \\
(\mathrm{Gyr})\end{array}$ & Reference & {$[\mathrm{Fe} / \mathrm{H}]$} & Reference \\
\hline \multicolumn{6}{|c|}{ SMC } \\
\hline NGC $411 \ldots \ldots \ldots$ & $\mathrm{V}-\mathrm{VI}$ & 1.8 & 11 & -0.84 & 11 \\
\hline NGC $416 \ldots \ldots \ldots$ & VI & 6.9 & 11 & -1.44 & 11 \\
\hline NGC 419 ......... & $\mathrm{V}$ & 1.2 & 11 & -0.70 & 11 \\
\hline \multicolumn{6}{|c|}{ LMC } \\
\hline NGC $1651 \ldots \ldots$. & V & 1.6 & 12 & -0.37 & 1 \\
\hline NGC $1751 \ldots \ldots$ & VI & 1.5 & 10 & -0.18 & 1 \\
\hline NGC $1754 \ldots \ldots .$. & VII & 15.5 & 11 & -1.42 & 11 \\
\hline NGC $1777 \ldots \ldots .$. & IV B & 0.76 & 7 & -0.35 & 1 \\
\hline NGC $1783 \ldots \ldots$. & $\mathrm{V}$ & 0.87 & 10 & -0.75 & 3 \\
\hline NGC $1795 \ldots \ldots .$. & V & 1.7 & 8 & -0.23 & 1 \\
\hline NGC $1806 \ldots \ldots .$. & V & 0.50 & 12 & -0.23 & 1 \\
\hline NGC $1818 \ldots \ldots . .$. & I & 0.04 & 10 & -0.9 & 4 \\
\hline NGC $1831 \ldots \ldots$. & IV A & 0.37 & 9 & +0.01 & 1 \\
\hline NGC $1846 \ldots \ldots .$. & VI & 1.2 & 10 & -0.70 & 1 \\
\hline NGC $1866 \ldots \ldots .$. & III & 0.22 & 9 & -1.2 & 4 \\
\hline NGC $1868 \ldots \ldots .$. & IV A & 0.45 & 9 & -0.50 & 1 \\
\hline NGC 1978....... & VI & 1.7 & 10 & -0.42 & 1 \\
\hline NGC $2010 \ldots \ldots .$. & III & 0.16 & 9 & +0.0 & 5 \\
\hline NGC $2133 \ldots \ldots$. & IV A & 0.13 & 5 & -1.0 & 5 \\
\hline NGC $2134 \ldots \ldots .$. & III & 0.19 & 9 & -1.0 & 5 \\
\hline NGC $2136 \ldots \ldots$. & III & 0.11 & 10 & -0.4 & 4 \\
\hline NGC $2155 \ldots \ldots .$. & VI & 2.8 & 10 & -0.55 & 1 \\
\hline NGC $2162 \ldots \ldots$. & $\mathrm{V}$ & 0.74 & 9 & -0.23 & 1 \\
\hline NGC $2164 \ldots \ldots .$. & II & 0.10 & 9 & -0.6 & 5 \\
\hline NGC $2173 \ldots \ldots$. & VI & 1.7 & 10 & -0.24 & 1 \\
\hline NGC $2190 \ldots \ldots$ & $\mathrm{V}$ & 0.74 & 9 & -0.12 & 1 \\
\hline NGC $2193 \ldots \ldots$. & $\mathrm{V}$ & 2.0 & 7 & -0.5 & 5 \\
\hline NGC $2203 \ldots \ldots .$. & VI & 1.8 & 8 & -0.52 & 1 \\
\hline NGC $2210 \ldots \ldots .$. & VII & 12.3 & 11 & -1.97 & 1 \\
\hline NGC $2213 \ldots \ldots .$. & $\mathrm{V}$ & 1.0 & 10 & -0.01 & 1 \\
\hline NGC $2214 \ldots \ldots .$. & II & 0.08 & 9 & -1.2 & 5 \\
\hline NGC $2249 \ldots \ldots$. & IV B & 0.52 & 9 & -0.05 & 4 \\
\hline
\end{tabular}

a SWB types taken from Bica et al. 1996.

ReFEREnCeS.- (1) Olszewski et al. 1991; (2) Olsen et al. 1999; (3) Cohen 1982; (4) Seggewiss \& Richtler 1989; (5) Sagar \& Pandey 1989; (6) Durand, Hardy, \& Melnick 1984; (7) Elson \& Fall 1988; (8) Geisler et al. 1997; (9) Girardi et al. 1995; (10) Girardi \& Bertelli 1998; (11) Piatti et al. 2002; (12) Dirsch et al. 2000.

a serious underestimate of the true Balmer line strengths in the young populations, where much of the $\mathrm{EW}$ is in the extended line wings. On the other hand, using broad bandpasses introduces a great deal of contamination from other features in the case of the older populations and is not well suited to the weak metal lines in the younger populations.

An alternative strategy is to focus on obtaining a measure of the central line depths, where the particular feature of interest achieves its greatest contrast with other "contaminating" features. An approach formulated by Rose (1984, $1985,1994)$ is to leverage a Balmer line center against a neighboring metal feature. Specifically, an index is defined by taking the ratio of the counts in the bottoms of two neighboring spectral absorption features, without reference to the continuum levels. The disadvantages of this approach are twofold. First, the index loses sensitivity if one of the features becomes saturated in the line core and also becomes more difficult to measure because of the lower signalto-noise ratio $(\mathrm{S} / \mathrm{N})$ in the line center with increasing line depth. Second, all indices represent relative measurements of one feature against another, while a true EW measurement of a single feature is ideal for resolving the degeneracy between age and metallicity. A key advantage of line ratio indices is in maximizing the contribution of the particular spectral lines, in the face of confusion from other features, by working in the deepest part of the lines. In addition the index is defined without requiring knowledge of the often problematic location of the (pseudo) continuum. Because neighboring absorption features are used, the measured indices are virtually insensitive to reddening and to errors in spectrophotometry. Moreover, since both line centers tend to weaken equally with decreasing spectral resolution, the indices are only mildly sensitive to spectral broadening. To summarize, while we make extensive use of line ratio indices in this paper, there are indeed other means of characterizing the same key features that we utilize.

\subsection{Line Ratio Indices}

The age-dating technique presented in this paper is primarily based on two of the spectral indices developed and calibrated in Rose $(1984,1985)$ and explicitly modeled in LR96 and in Leonardi \& Worthey (2000). The specific features used, along with the Balmer discontinuity index passbands (see $\S 3.3$ ), are identified in the synthetic spectrum of a star (generated from the Kurucz 1993 SYNTHE code, as described in $\S 4.2$ ) in Figure 2. The first spectral index (hereafter referred to as the $\mathrm{H} \delta / \lambda 4045$ index) is calculated by taking the ratio of the residual central intensity in $\mathrm{H} \delta$ relative to that of the neighboring $\mathrm{Fe}$ i $\lambda 4045$ line. The absorption-line indices are calculated by finding the minimum pixel value in a small range around the line center for each of the two lines and then forming the appropriate ratio. Because of the manner in which the index is defined, it decreases in value as one proceeds from late-type stars to earlier-type stars, i.e., as $\mathrm{H} \delta$ strengthens and $\mathrm{Fe}$ I $\lambda 4045$ weakens. It is essentially a measure of the spectral type of a

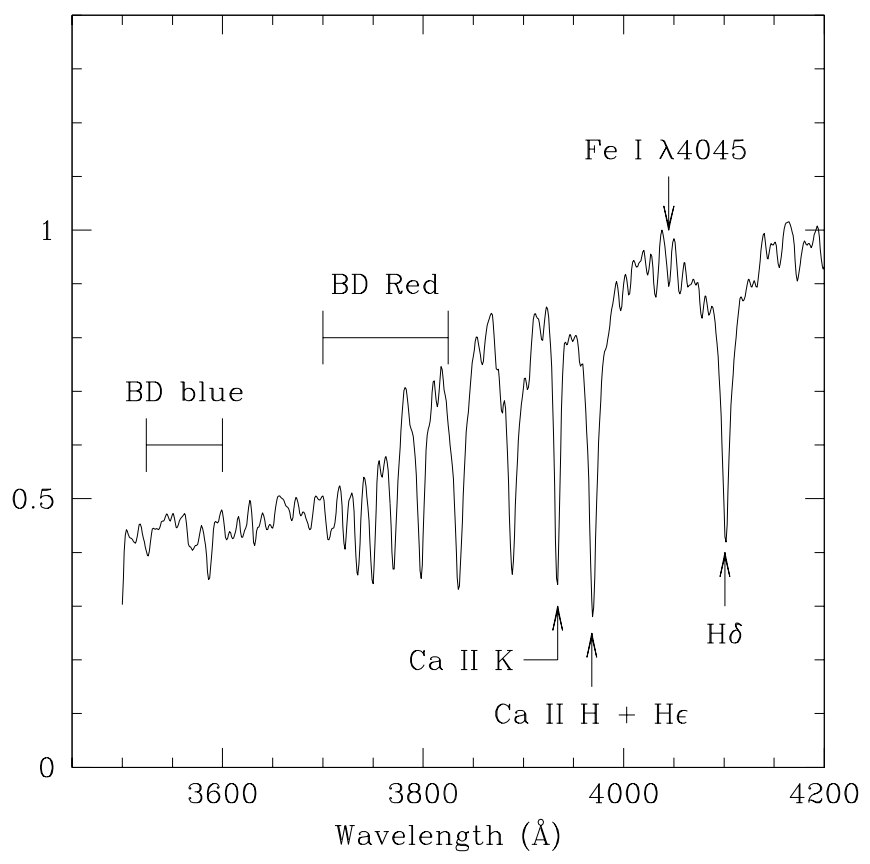

FIG. 2.- Synthetic spectrum of a star with $T_{\text {eff }}=7500 \mathrm{~K}, \log g=5.0$, and solar chemical composition plotted with all spectral features identified that are used in this paper. 
star (or of the integrated spectral type of a stellar population). The $\mathrm{H} \delta / \lambda 4045$ index decreases smoothly from spectral type $\mathrm{K}$ through $\mathrm{A} 0$, where it reaches a minimum value. For spectral types earlier than $\mathrm{A} 0, \mathrm{H} \delta / \lambda 4045$ increases again, due to the weakening of the Balmer lines in $\mathrm{B}$ and $\mathrm{O}$ stars and the virtual disappearance of $\mathrm{Fe}$ I $\lambda 4045$. The integrated spectral type as measured by the $\mathrm{H} \delta / \lambda 4045$ index will serve as the baseline for the age/metallicity determination method.

The second index, formed from the ratio of the $\mathrm{Ca}$ II $\mathrm{H}+\mathrm{H} \epsilon$ line relative to $\mathrm{Ca}$ II $\mathrm{K}$ (and hereafter referred to as the $\mathrm{Ca}$ II index) behaves quite differently, due to the fact that $\mathrm{Ca}$ II $\mathrm{H}+\mathrm{H} \epsilon$ is dominated by $\mathrm{Ca}$ II $\mathrm{H}$ for cooler stars and by $\mathrm{H} \epsilon$ for hotter stars. Due to saturation of the $\mathrm{Ca}$ II $\mathrm{H}$ and $\mathrm{K}$ line cores, it has a constant value for stars with a spectral type later than about F5. This constant value equals $\sim 1.2$ and $\sim 1.3$ for dwarf stars and giant stars, respectively. The behavior of $\mathrm{Ca}$ II, when plotted against $\mathrm{H} \delta / \lambda 4045$ (as a surrogate for spectral type) is illustrated in Figure 3 for a sample of 684 stars covering a wide region of $T_{\text {eff, }} \log g$, and $[\mathrm{Fe} / \mathrm{H}]$. There it can be seen that for $\mathrm{H} \delta / \lambda 4045 \geq 0.7$ (approximately spectral type F5), the Ca II indices are flat for all later-type dwarfs and giants. For earlier-type stars, however, the index decreases dramatically as the $\mathrm{Ca}$ II lines weaken and $\mathrm{H} \epsilon$ strengthens. As with $\mathrm{H} \delta / \lambda 4045$, it reaches a minimum at spectral type $\mathrm{A} 0$ and then increases toward earlier spectral types as $\mathrm{H} \epsilon$ fades at higher temperatures. The constant value of $\mathrm{Ca}$ II in late $\mathrm{F}-\mathrm{K}$ stars and the sudden drop at earlier types provides an unambiguous signature for

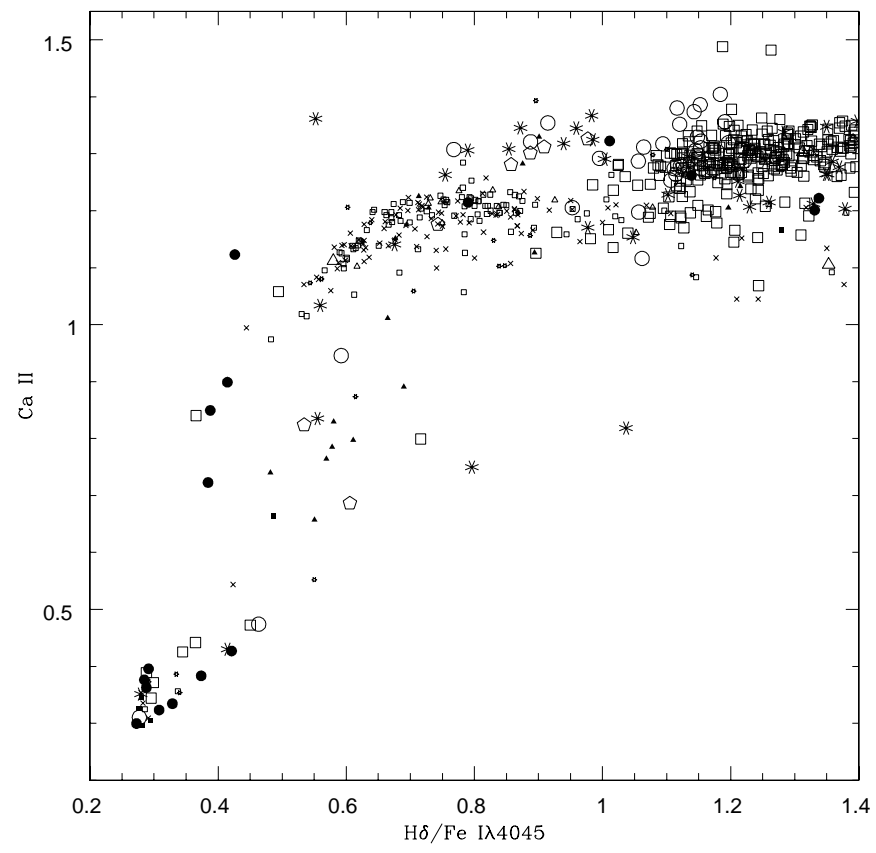

FIG. 3.- Ca II index plotted vs. the $\mathrm{H} \delta / \mathrm{Fe}$ I $\lambda 4045$ index for stars in the CFSL. Different symbols denote stars in various gravity and metallicity bins. Large filled circles are for stars with intermediate gravity of $3.3<\log g \leq 3.7$. Large octagonal asterisks are for low-gravity (supergiant) stars with $1.8 \geq \log g$. Giant stars $(1.8<\log g \leq 3.3)$ are plotted as large open symbols, with triangles, squares, circles, and pentagons referring, respectively, to the following metallicity intervals: $[\mathrm{Fe} / \mathrm{H}]>+0.3$; $+0.3 \geq[\mathrm{Fe} / \mathrm{H}]>-0.4 ; \quad-0.4 \geq[\mathrm{Fe} / \mathrm{H}]>-1.5 ;-1.5 \geq[\mathrm{Fe} / \mathrm{H}]$. Dwarfs are plotted as small symbols, with the following scheme: filled squares for $[\mathrm{Fe} / \mathrm{H}]>+0.3$; times crosses for $+0.3 \geq[\mathrm{Fe} / \mathrm{H}]>-0.2$; open squares for $-0.2>[\mathrm{Fe} / \mathrm{H}]>-0.75$; open triangles for $-0.75>[\mathrm{Fe} / \mathrm{H}]>-1.25$; filled triangles for $-1.25 \geq[\mathrm{Fe} / \mathrm{H}]$.

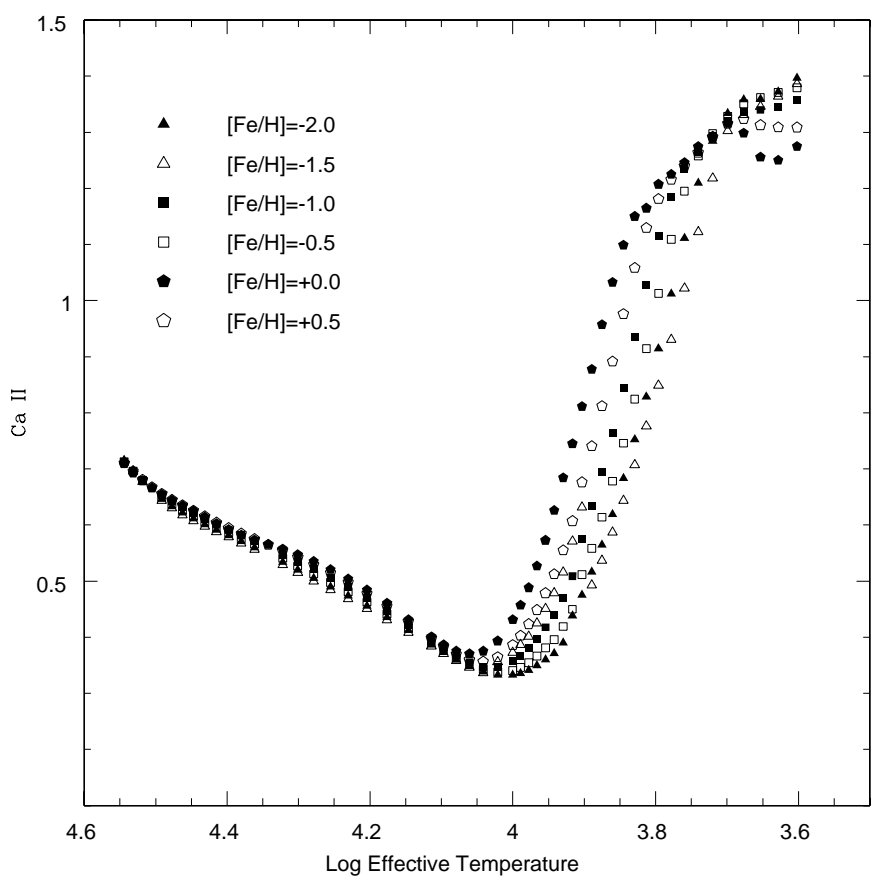

FIG. 4.-The Ca II index plotted vs. $\log \left(T_{\text {eff }}\right)$ for synthetic dwarf stars. Note the separation in metallicity for intermediate $T_{\text {eff }}$.

stars hotter than F5 in the integrated light of a stellar population if the index value falls below the constant value for cool stars. Since the MS turnoff is likely to be the major contributor to the integrated light in the visible part of the spectrum, Ca II can in principle be used as a YSP age indicator up to the MS lifetime of an F5 star, or approximately 4 Gyr. In addition, the $T_{\text {eff }}$ at which the Ca II index begins its sharp drop is a function of $[\mathrm{Fe} / \mathrm{H}]$. This is illustrated for dwarf stars in Figure 4, where the $\mathrm{Ca}$ II index, as derived from a large sample of synthetic stellar spectra (described in $\S 4.2$ ), is plotted versus $T_{\text {eff. }}$. For the low-metallicity stars, the drop in Ca II begins already at $\log \left(T_{\text {eff }}\right) \sim 3.7\left(T_{\text {eff }} \sim 5000 \mathrm{~K}\right)$, while for metal-rich stars the drop begins close to $\log \left(T_{\text {eff }}\right) \sim 3.85\left(T_{\text {eff }} \sim 7000 \mathrm{~K}\right)$. Consequently, the detailed behavior of the integrated $\mathrm{Ca}$ II index has a metallicitydependent component. Finally, it should be noted that in active star-forming regions, the behavior described above is no longer valid because the $\mathrm{H} \epsilon$ and $\mathrm{H} \delta$ absorption lines can be contaminated by emission fill-in.

\subsection{Balmer Discontinuity Index}

Since it was determined in LR96 that chemical composition may play a significant role in deriving the integrated age of a poststarburst stellar population, another index is required to fully describe the age-strength-metallicity parameter space. The Balmer discontinuity (BD) is an effective age indicator for young star clusters (e.g., Bica et al. 1990) and will serve as the third index. We define a BD index as the ratio of the average flux in the wavelength interval $3700-3825 \AA$ to that in the interval $3525-3600 \mathrm{~A}$, following Rose, Stetson, \& Tripicco (1987). The BD index contains a great deal of dynamic range for hot stars, evolving rapidly as a function of spectral type. As in the case of the two absorption-line ratio indices, the $\mathrm{BD}$ index is based on Balmer transitions (in this case the discontinuity at $\sim 3650 \AA$ produced by the bound-free transition); thus, it has an 


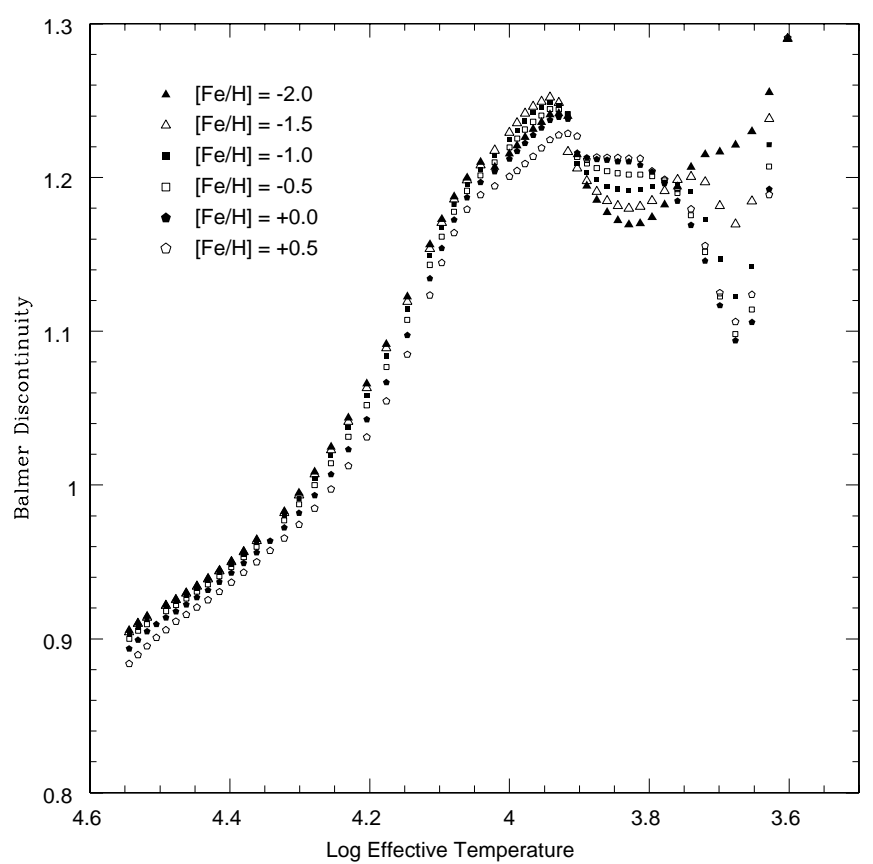

FIG. 5.-Balmer discontinuity index plotted vs. $\log \left(T_{\text {eff }}\right)$ for synthetic dwarf stars. The steep dependence on $T_{\text {eff }}$ makes the BD index a very good age indicator for young populations.

extreme value at spectral type A0, where the Balmer lines are strongest and falls off to both earlier and later types. The behavior of the BD index with $\log T_{\text {eff }}$ is shown in Figures 5 and 6. Of particular note is the strong increase in the $\mathrm{BD}$ index with decreasing gravity, i.e., opposite to the trend in the Balmer lines. With all three indices in this paper based on Balmer transitions, they are partially redundant in their ability to distinguish stellar populations. However,

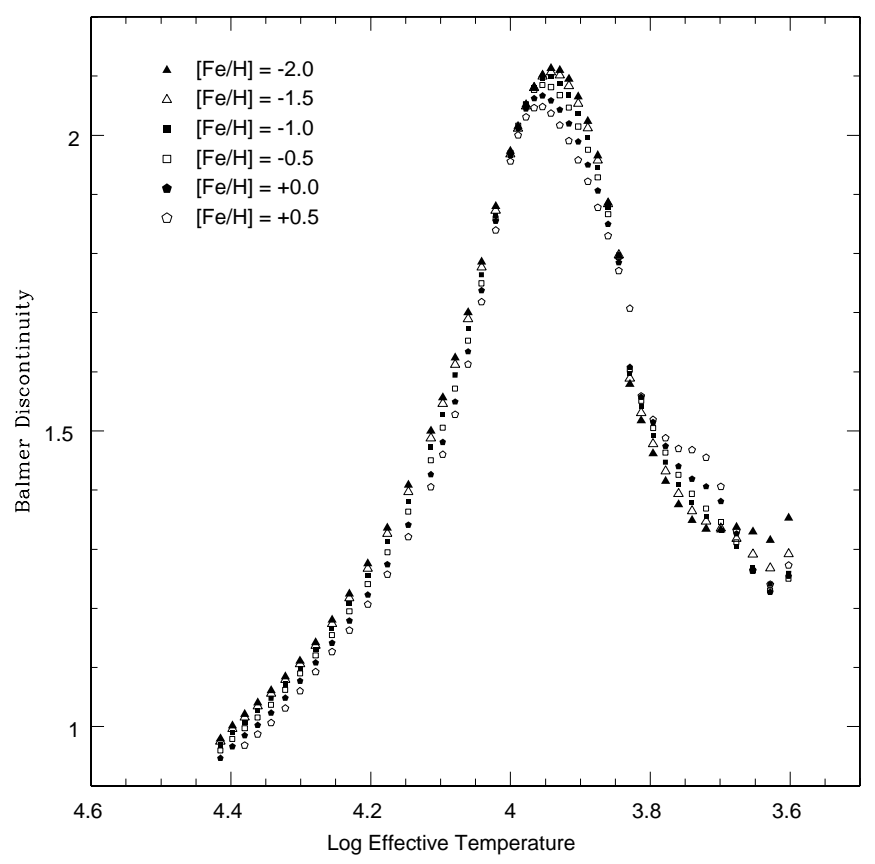

FIG. 6.-Balmer discontinuity index plotted vs. $\log \left(T_{\text {eff }}\right)$ for synthetic giant stars. there is enough difference in behavior between them to act effectively in combination.

\subsection{Cluster Index Errors}

Since most clusters were observed only once, the errors were computed by using photon statistics for each spectral feature in the absorption feature indices. The indices used in the age-dating procedure are calculated from cluster spectra that have been Gaussian-smoothed from their original 3.2 $\AA$ (FWHM) resolution, with a $\sigma$ of $2.0 \AA$. Hence, the number of counts used to formulate the photon statistics were calculated by performing a weighted sum of the counts in the central pixel and 4 pixels on either side for each of the index line feature components in the raw cluster spectra. A normalized Gaussian with the same width as the smoothing Gaussian was used as the weighting function. The number of counts resulting from the sum is equivalent to the counts in the central pixel of the line feature in the cluster spectrum after smoothing. This value was combined in quadrature with the read noise to obtain the total error in the measurement for the residual central line intensity for each absorption feature in the smoothed spectra. Errors for the BD index bandpasses were calculated by taking the square root of the average number of counts in each bandpass and dividing by the width of the bandpass. The effects of smoothing on the $\mathrm{BD}$ index errors and on the index itself were deemed negligible.

The above errors are based solely on photon statistics. Systematic errors may be present as well. In fact, as will be seen in $\S 6$, there is a discrepancy of $\sim 0.1$ between the BD index values predicted by the models described in $\S 5$ and the observed values of many of the clusters. The possibility that the discrepancy could lie in the evolutionary synthesis models and/or the synthetic spectra used as inputs for those models will be considered later. Here we evaluate the likelihood of systematic errors in the observations, specifically in background subtraction and in spectrophotometry.

In regard to improper background subtraction affecting the observed $\mathrm{BD}$ indices, the background measurements were taken from the edges of the slit in the cluster observations, rather than from separate sky exposures, and the average signal-to-background ratio at $3600 \AA$ is $\sim 2.1$, ranging from a minimum of 0.39 for NGC 2190 to a maximum of 4.9 for NGC 416 . These values are certainly low enough to render errors in the background subtraction a real possibility. However, the likelihood that background errors play an important role is seriously undercut by noting the location of the BD index for 47 Tuc (see Fig. 18). The BD index for 47 Tuc is $\sim 0.06$ directly below its proper age point for its accepted metallicity of $[\mathrm{Fe} / \mathrm{H}]=-0.7$, yet the clusterto-background ratio is 9.5. If background subtraction problems were the principle issue, one would expect to see an inverse correlation between the deviation of models from data and the cluster-to-background ratio. However, 47 Tuc presents a striking counterexample to such a hypothesis.

Errors in spectrophotometry could also account for the outlying clusters. The main emphasis of the cluster observations was on obtaining the absorption feature indices; hence, only two flux calibration stars were observed at the beginning and end of each night. With the outlying clusters departing from the models by only $\sim 0.1$ in the $\mathrm{BD}$ index, an error of $\sim 10 \%$ in the relative spectrophotometry across the wavelength region $3500-3800 \AA$ can account for the 
discrepancy. Achieving 10\% accuracy in spectrophotometry at $3500 \AA$ is ordinarily quite challenging, requiring more than a couple of spectrophotometric measurements. On the other hand, the principal problem for accurate spectrophotometry in the blue, differential atmospheric refraction, is probably not an issue for our observations. While the slit was not rotated to the parallactic angle, in the case of the spectrophotometric standards we used a wide slit, and in the case of the clusters they are all extended objects; and the spectrograph slit was trailed at a uniform rate over the core diameter of the cluster, so that the atmospheric dispersion effect is avoided. Another potential problem for our spectrophotometry is that we used the standard CTIO extinction curve, thus leaving us vulnerable to possible systematic differences in extinction during our run, when compared with the standard curve. We note that atmospheric extinction tends to be particularly variable in the near-UV.

We do have a limited number of stars observed that have reasonably well-defined atmospheric parameters. These stars, however, were observed through the narrow slit and not at the parallactic angle, and thus are subject to all of the uncertainties regarding atmospheric dispersion. Overall, due to the steep dependence of the BD index on both $T_{\text {eff }}$ and $\log g$, coupled with the uncertainties in the fundamental determinations of these parameters for the limited number of observed stars and with the possibility of spectrophotometric errors introduced by atmospheric dispersion, we cannot determine at the $10 \%$ level whether the observed stars agree with the synthetic spectrum values or not. One additional handle on the situation is that, if spectrophotometric errors are involved, one might expect that the errors would be particularly severe on one night. However, the clusters with the discrepancy in BD index between observations and models were observed over several nights.

In conclusion, we cannot rule out systematic errors in the $\mathrm{BD}$ indices of the clusters at the $10 \%$ level. However, the above discussion leads us to suspect that discrepancies between data and models at that level are more likely due to the models themselves, rather than to observational errors in the cluster indices.

\section{SPECTRAL LIBRARY}

Modeling stellar populations requires building up the integrated properties of the population from the individual, well-determined properties of its components. To do so, an extensive spectral library (for integrated spectroscopic properties) is needed as building blocks for the population models. The ideal library is composed of high $\mathrm{S} / \mathrm{N}$, highresolution stellar spectra, obtained with a single stable telescope/spectrograph/detector combination, which fully sample all possible stellar atmospheric parameters and stages of stellar evolution. In reality, because of the limitations imposed by the particular star formation and chemical enrichment history of our Galaxy, certain areas of the parameter space are not represented in the solar neighborhood. In particular, for the young systems we are interested in, the hot stars in the spectral library must be well represented across a large metallicity range. Given the lack of hot, metal-poor, high-gravity stars in the Galaxy, we cannot hope to generate adequate coverage of YSPs using a purely empirical spectral library. On the other hand, as is further discussed in $\S 4.3$, correctly reproducing the detailed absorption spectra in cool stars is challenging, and so the use of empirical spectra is favored. Thus, in modelling YSPs of all chemical compositions, we found it is necessary to assemble a spectral library containing both empirical stellar spectra and synthetic spectra.

\subsection{Empirical Spectra}

The W94 models utilize polynomial functions derived from fits to stellar spectral features in the spectral library. The fitting functions express how the stellar features vary as a function of effective temperature, gravity, and metallicity. Consequently, they require spectra of stars with well-determined atmospheric parameters covering a wide range of the parameter space. For our purposes, we also require highresolution spectra to accurately measure the absorption-line indices. Such a library was compiled by Jones (1999; see also Leitherer et al. 1996) at the KPNO coudé feed telescope ${ }^{3}$ (this homogeneous library of stellar spectra is hereafter referred to as the Coudé Feed Spectral Library [CFSL]). The CFSL consists of two spectra, a red one and a blue one, for each of 684 stars. We limit our discussion here to only the blue spectra. These spectra cover the wavelength range $3820-4509 \AA$ and have a pixel sampling of $0.622807 \AA$ pixel $^{-1}$ with a spectral resolution of $1.8 \AA$ FWHM. The library is well populated at low effective temperatures in a large metallicity range and covering a suitable range in surface gravity. But at higher temperatures the coverage is limited, primarily as a result of the lack of hot, metal-poor stars. To insure adequate sampling throughout the metallicity range, we restrict the effective temperature range for the empirical library to be $4350-6300 \mathrm{~K}$. Of the stars in this range, some were eliminated either for having chromospheric emission, which contaminates the absorption-line indices (Rose 1984), or for having uncertain atmospheric parameters, which includes both undetermined parameters and parameters that clearly do not agree with their measured indices. In all, 523 CFSL stars are included in the empirical spectral library used for the models.

To produce the fitting functions, we employ a fitting program kindly provided by G. Worthey. It uses a series of least-squares regressions to fit a third-order polynomial in $\Theta\left(=5040 / T_{\text {eff }}\right),[\mathrm{Fe} / \mathrm{H}]$, and $\log g$. In an iterative procedure the terms in the polynomial are either included or excluded until no systematic trends appear in the residuals of the atmospheric parameters versus the polynomial fit. Each absorption line used in the spectral indices (e.g., $\mathrm{H} \delta$ ) is fitted with one polynomial across the entire temperature range. The coefficients for the adopted polynomials are given in Table 3. Before the fitting procedure the stellar spectra were normalized to unity at $4000 \AA$ and rebinned to $1 \AA$ pixel $^{-1}$ dispersion. Unfortunately, the BD index could not be fitted, since the empirical spectra do not go far enough into the blue. Hence, all results and conclusions derived from the BD index are based solely on the synthetic spectral library described below.

\subsection{Synthetic Spectra}

The CFSL has complete metallicity coverage only for temperatures cooler than $7000 \mathrm{~K}$ because of the aforementioned lack of young, metal-poor stars in the Galaxy. To

\footnotetext{
${ }^{3}$ The spectra are available from the NOAO FTP archive at ftp://ftp.noao.edu/catalogs/coudelib.
} 
TABLE 3

Empirical Polynomial Coefficients

\begin{tabular}{|c|c|c|c|c|}
\hline Parameter & $\mathrm{Ca}$ II $\mathrm{K}$ & $\mathrm{Ca}$ II $\mathrm{H}$ & $\mathrm{Fe}_{\mathrm{I}} \lambda 4045$ & $\mathrm{H} \delta$ \\
\hline 1. Constant & 0.3522 & 0.3862 & 2.5286 & -3.1050 \\
\hline 2. $\Theta$. & -0.2541 & -0.2606 & -2.7922 & 7.2148 \\
\hline 3. $\Theta^{2}$.. & $\ldots$ & $\ldots$ & 1.0882 & -3.2809 \\
\hline 4. $\Theta^{3}$. & .. & .. & $\ldots$ & 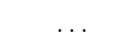 \\
\hline 5. $[\mathrm{Fe} / \mathrm{H}] .$. & -2.6599 & -0.3271 & $\ldots$ & 0.1577 \\
\hline 6. $[\mathrm{Fe} / \mathrm{H}]^{2} \ldots .$. & 0.02995 & 0.01763 & 0.01450 & $\ldots$ \\
\hline 7. $[\mathrm{Fe} / \mathrm{H}]^{3} \ldots \ldots \ldots \ldots \ldots \ldots$ & 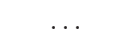 & & $\ldots$ & 0.004024 \\
\hline 8. $(\log g) \ldots \ldots$ & 0.01866 & 0.02340 & $\ldots$ & $\ldots$ \\
\hline 9. $(\log g)^{2}$. & $\ldots$ & $\ldots$ & $\ldots$ & $\ldots$ \\
\hline 10. $(\log g)^{3}$. & $\ldots$ & $\ldots$ & $\ldots$ & $\ldots$ \\
\hline 11. $(\Theta)([\mathrm{Fe} / \mathrm{H}]) \ldots \ldots \ldots \ldots$ & 4.9594 & 0.2794 & -0.0862 & -0.1500 \\
\hline 12. $(\Theta)([\mathrm{Fe} / \mathrm{H}])^{2} \ldots \ldots \ldots \ldots$ & $\cdots$ & $\cdots$ & $\cdots$ & $\cdots$ \\
\hline 13. $(\Theta)(\log g) \ldots \ldots \ldots \ldots \ldots$ & $\ldots$ & $\ldots$ & $\ldots$ & $\ldots$ \\
\hline 14. $(\Theta)(\log g)^{2} \ldots \ldots \ldots \ldots \ldots$ & $\ldots$ & & 0.005235 & 0.002516 \\
\hline 15. $([\mathrm{Fe} / \mathrm{H}])(\log g) \ldots \ldots .$. & 0.01524 & 0.01166 & $\ldots$ & $\ldots$ \\
\hline 16. $([\mathrm{Fe} / \mathrm{H}])(\log g)^{2} \ldots \ldots$ & & $\ldots$ & $\ldots$ & $\ldots$ \\
\hline 17. $([\mathrm{Fe} / \mathrm{H}])(\Theta)^{2} \ldots \ldots \ldots \ldots$ & -2.3205 & $\cdots$ & $\ldots$ & $\ldots$ \\
\hline 18. $(\log g)([\mathrm{Fe} / \mathrm{H}])^{2} \ldots \ldots$ & $\cdots$ & $\ldots$ & $\ldots$ & $\ldots$ \\
\hline 19. $(\log g)(\Theta)^{2} \ldots \ldots \ldots \ldots \ldots$ & $\ldots$ & $\ldots$ & $\ldots$ & $\ldots$ \\
\hline
\end{tabular}

remedy this shortcoming, as well as to supply wavelength coverage down to $3500 \AA$ for the BD index, the spectral library was augmented with 2095 synthetic spectra. These spectra were generated from ATLAS model atmospheres (Kurucz 1994) and the SYNTHE synthetic spectrum codes (Kurucz 1993) that were kindly supplied by R. Kurucz (1995, private communication).

The SYNTHE program generates a synthetic spectrum from a given model stellar atmosphere and a spectral line list. The model atmospheres are generated from Kurucz's (1994) ATLAS program, and a packaged set of 2095 of those atmospheres was taken from the available CD-ROM provided by R. Kurucz. The SYNTHE program is actually a series of VMS FORTRAN programs that were converted to run on a SUN/UNIX workstation. A stellar spectrum was computed for every available stellar atmosphere contained on the CD-ROM of Kurucz (1994). For reasons of available time, ATLAS was not itself used to increase the number of stellar atmospheres.
The spectral line list is the same for each computed spectrum. Rather than individually assessing which species are important for a particular set of stellar atmospheric parameters, every species is included for every star. These include $\mathrm{Fe}, \mathrm{CN}, \mathrm{C}_{2}, \mathrm{CO}, \mathrm{SiO}$, and $\mathrm{H}_{2}$. The SYNTHE CD-ROM has a series of data files that contain the line list information for the atomic and molecular species. Once the line list was generated, the basic parameters for the spectra were selected. Specifically, the wavelength range was chosen to be 3500 $5500 \AA$ and the resolution is $\lambda / \Delta \lambda=72,700$. The default microturbulent velocity of $2.0 \mathrm{~km} \mathrm{~s}^{-1}$ was used for each spectrum.

The final atmospheric parameter space covered by the grid is given in Table 4. The calculated spectral indices of the SYNTHE spectra vary systematically as a function of the atmospheric parameters and contain no observational error. Hence, instead of applying fitting functions to the variation in spectral indices with atmospheric parameters, we linearly interpolate between the line strengths of the synthetic spectra to find line strengths for a given set of atmospheric parameters between the grid points.

Although we have greatly extended our ability to model YSPs with the addition of the synthetic spectra, the theoretical HR diagram is still not covered completely. Specifically, the sampling suffers for very hot giant stars, which make significant contributions to the integrated light of very young $(<0.1 \mathrm{Gyr})$ stellar populations, especially in the blue part of the spectrum. With few library spectra, synthetic or empirical, near these points in atmospheric parameter space, interpolation within the grid at these points or extrapolation beyond it needs to be handled with care.

As seen in Table 4, there are no spectra with $\log g<2.0$ for all $T_{\text {eff, }}, \log g<3.0$ for $T_{\text {eff }}>10,500 \mathrm{~K}$, and $\log g<4.0$ for $T_{\text {eff }}>26,000 \mathrm{~K}$. We consider temperature extrapolation of the spectral indices beyond either of the synthetic grid absolute endpoints, $T_{\text {eff }}<4000 \mathrm{~K}$ or $T_{\text {eff }}>35,000 \mathrm{~K}$, to be highly uncertain, and we ignore all contributions from such stars. In other cases, e.g., for hot giants, we use the following scheme: We do not have enough spectra to bracket the isochrone points in temperature, so we drop down in temperature to the next nearest spectrum to maintain the ability to interpolate. There is still the need to have spectra on each side of the isochrone point in all three atmospheric

TABLE 4

Stellar Atmospheric Parameter Space for SYNTHE

\begin{tabular}{|c|c|c|c|c|c|}
\hline $\begin{array}{l}T_{\text {eff }} \\
(\mathrm{K})\end{array}$ & $\begin{array}{l}\Delta T \\
(\mathrm{~K})\end{array}$ & $\log g$ & $\Delta(\log g)$ & {$[\mathrm{Fe} / \mathrm{H}]$} & $\Delta([\mathrm{Fe} / \mathrm{H}])$ \\
\hline $4000-9750 \ldots \ldots \ldots \ldots \ldots \ldots$ & 250 & $2.0-5.0$ & 1.0 & $\begin{array}{l}-2.0 \text { to }-0.5 \\
-0.3-0.3 \\
0.5\end{array}$ & $\begin{array}{l}0.5 \\
0.1\end{array}$ \\
\hline $10000-10500$............... & 500 & $2.0-5.0$ & 1.0 & $\begin{array}{l}-2.0 \text { to }-0.5 \\
-0.3-0.3 \\
0.5\end{array}$ & $\begin{array}{l}0.5 \\
0.1\end{array}$ \\
\hline $11000-13000$................. & 500 & $3.0-5.0$ & 1.0 & $\begin{array}{l}-2.0 \text { to }-0.5 \\
-0.3-0.3 \\
0.5\end{array}$ & $\begin{array}{l}0.5 \\
0.1\end{array}$ \\
\hline $14000-26000 \ldots \ldots \ldots \ldots \ldots$ & 1000 & $3.0-5.0$ & 1.0 & $\begin{array}{l}-2.0 \text { to }-0.5 \\
-0.3-0.3 \\
0.5\end{array}$ & $\begin{array}{l}0.5 \\
0.1\end{array}$ \\
\hline $27000-35000 \ldots \ldots \ldots \ldots \ldots$ & 1000 & $4.0-5.0$ & 1.0 & $\begin{array}{l}-2.0 \text { to }-0.5 \\
-0.3-0.3 \\
0.5\end{array}$ & $\begin{array}{l}0.5 \\
0.1\end{array}$ \\
\hline
\end{tabular}


TABLE 5

SAMPle INTERPOLATION SCHEME

\begin{tabular}{|c|c|c|c|c|c|c|}
\hline \multicolumn{3}{|c|}{ IDEAL } & \multirow[b]{2}{*}{$\begin{array}{c}\text { PRESENT? } \\
\text { (4) }\end{array}$} & \multicolumn{3}{|c|}{ Actual } \\
\hline $\begin{array}{l}T_{\text {eff }}(\mathrm{K}) \\
\text { (1) }\end{array}$ & $\begin{array}{c}\log g \\
(2)\end{array}$ & $\begin{array}{c}{[\mathrm{Fe} / \mathrm{H}]} \\
(3)\end{array}$ & & $\begin{array}{l}T_{\mathrm{eff}}(\mathrm{K}) \\
\quad(5)\end{array}$ & $\begin{array}{c}\log g \\
(6)\end{array}$ & $\begin{array}{c}{[\mathrm{Fe} / \mathrm{H}]} \\
(7)\end{array}$ \\
\hline $16,000 \ldots \ldots \ldots \ldots \ldots$ & 2.0 & -1.0 & $\mathrm{~N}$ & 10500 & 2.0 & -1.0 \\
\hline $17,000 \ldots \ldots \ldots \ldots \ldots$ & 2.0 & -1.0 & $\mathrm{~N}$ & 10500 & 2.0 & -1.0 \\
\hline $16,000 \ldots \ldots \ldots \ldots \ldots$ & 3.0 & -1.0 & $\mathrm{Y}$ & 16000 & 3.0 & -1.0 \\
\hline $17,000 \ldots \ldots \ldots \ldots \ldots$ & 3.0 & -1.0 & $\mathrm{Y}$ & 17000 & 3.0 & -1.0 \\
\hline $16,000 \ldots \ldots \ldots \ldots \ldots$ & 2.0 & -0.5 & $\mathrm{~N}$ & 10500 & 2.0 & -0.5 \\
\hline $17,000 \ldots \ldots \ldots \ldots \ldots$ & 2.0 & -0.5 & $\mathrm{~N}$ & 10500 & 2.0 & -0.5 \\
\hline $16,000 \ldots \ldots \ldots \ldots \ldots$ & 3.0 & -0.5 & $\mathrm{Y}$ & 16000 & 3.0 & -0.5 \\
\hline $17,000 \ldots \ldots \ldots \ldots \ldots$ & 3.0 & -0.5 & $\mathrm{Y}$ & 17000 & 3.0 & -0.5 \\
\hline
\end{tabular}

a Isochrone point $-T_{\text {eff }}=16,300 \mathrm{~K}, \log g=2.4,[\mathrm{Fe} / \mathrm{H}]=-0.7$.

parameters. See Table 5 for an example of this interpolation scheme. For the given isochrone point, the first three columns are the library spectra that would be used for the interpolation if the synthetic grid were fully populated. The fourth column shows which spectra are not present in the library and the last three columns show the library spectra that are actually used in the model calculations. Since $T_{\text {eff }}=10,500 \mathrm{~K}$ is the hottest available spectrum with $\log g=2.0$, it serves as the lower interpolation in multiple instances. Its large distance from the isochrone point in temperature space, however, causes its actual weight to be small. It should be noted that this interpolation scheme only applies when $T_{\text {eff }}$ is hotter than $10,500 \mathrm{~K}$; hence, they only significantly affect the results for ages less than $\sim 0.1$ Gyr. The net effect is to produce some discontinuity in the index trajectories at the youngest ages, since some isochrone points remain excluded, but the probability of observing a star cluster or galaxy at such a young age is low.

\subsection{Empirical/SYNTHE Interface}

The reasons for combining the synthetic and empirical libraries to serve as the input library for the evolutionary synthesis models are compelling. The advantages of the synthetic spectra are threefold. First, this study is primarily concerned with YSPs, and the necessary observed stellar spectra in a wide metallicity range do not exist. Second, to take advantage of the age discrimination power of the $\mathrm{BD}$ index, we require regions of the spectrum that the empirical CFSL spectra do not reach. Finally, the increase in the HR diagram sampling is quite significant. On the other hand, keeping empirical spectra in the mix is not only satisfying, since they represent reality, but necessary, since the synthetic spectra diverge systematically from the CFSL spectra at low temperatures. In Figure 7 we plot the index values for both the synthetic and empirical stellar spectra at solar metallicity. The curves represent the synthetic spectra with $[\mathrm{Fe} /$ $\mathrm{H}]=0.0$, while the symbols represent the empirical stars in a small metallicity range around solar metallicity. In each set the spectra are grouped into gravity subsets. Overall, the agreement between the two libraries is quite good. There are two overt differences, however. First, the Ca II index for the dwarf empirical stars (squares) has a flatter slope than the synthetic curves for lower temperatures $(\mathrm{H} \delta / \lambda 4045>1.0)$. Second, the empirical giant stars (triangles) have a wider range of $\mathrm{Ca}$ II index values for a given $\mathrm{H} \delta / \lambda 4045$ index at cooler temperatures $(\mathrm{H} \delta / \lambda 4045 \sim 1.2)$ than the synthetic giants. At higher temperatures $(\mathrm{H} \delta / \lambda 4045<1.0)$, the libraries track very well, though there is a lack of hot giant stars. Similar behavior is seen at other metallicities as well, but there are significantly fewer empirical stars with which to make comparisons. Divergence at lower temperatures is not entirely unexpected, since the sheer abundance of metallic lines in real stars makes their spectra very complex. Including, with accuracy, every spectral feature of every species is extremely difficult.

To interface the two libraries, we chose a simple effective temperature cutoff. If the temperature prescribed by an isochrone is higher than $6000 \mathrm{~K}$, synthetic spectra are used in the calculations. If lower, the empirical fits are used. The cutoff temperature applies for all metallicities and gravities. Recall, however, that for the BD index, synthetic spectra are used at all effective temperatures. The interface is shown in

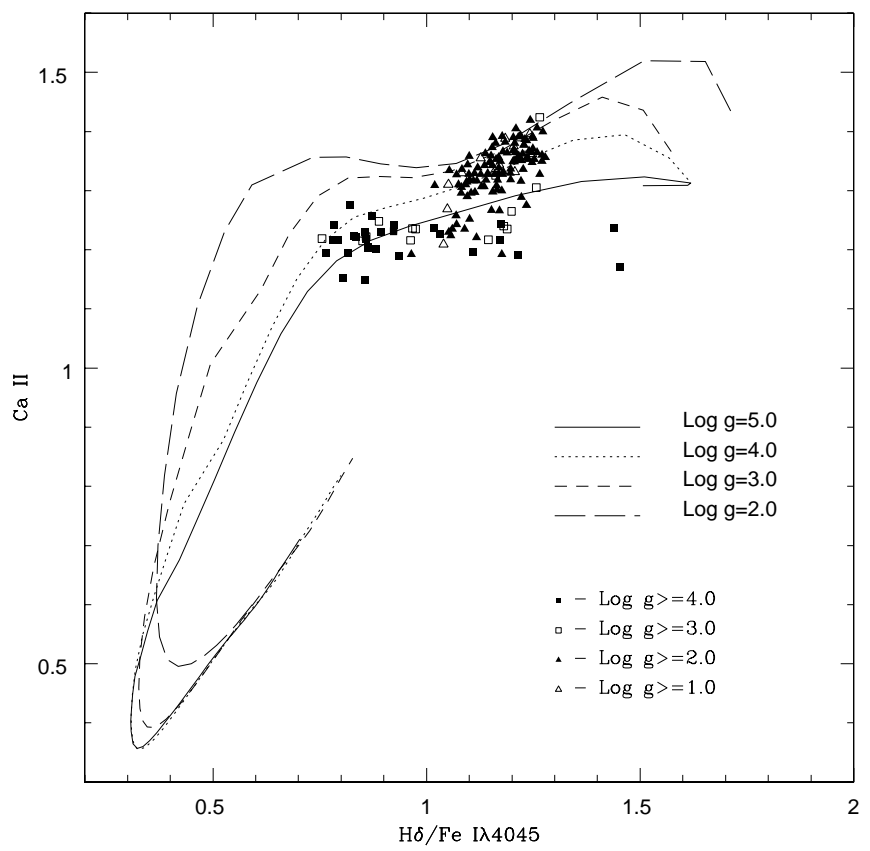

FIG. 7.- $\mathrm{H} \delta / \mathrm{Fe}$ I $\lambda 4045$ vs. Ca II (at solar metallicity) for both the empirical and synthetic libraries. Empirical stars are plotted with symbols, while the indices for the synthetic stars are plotted as lines. Both libraries have been further separated by gravity as shown in the figure legend. Notice that the empirical dwarfs have a flatter slope than the synthetic dwarfs as $\mathrm{H} \delta$ / Fe I $\lambda 4045$ increases past 1.0. 


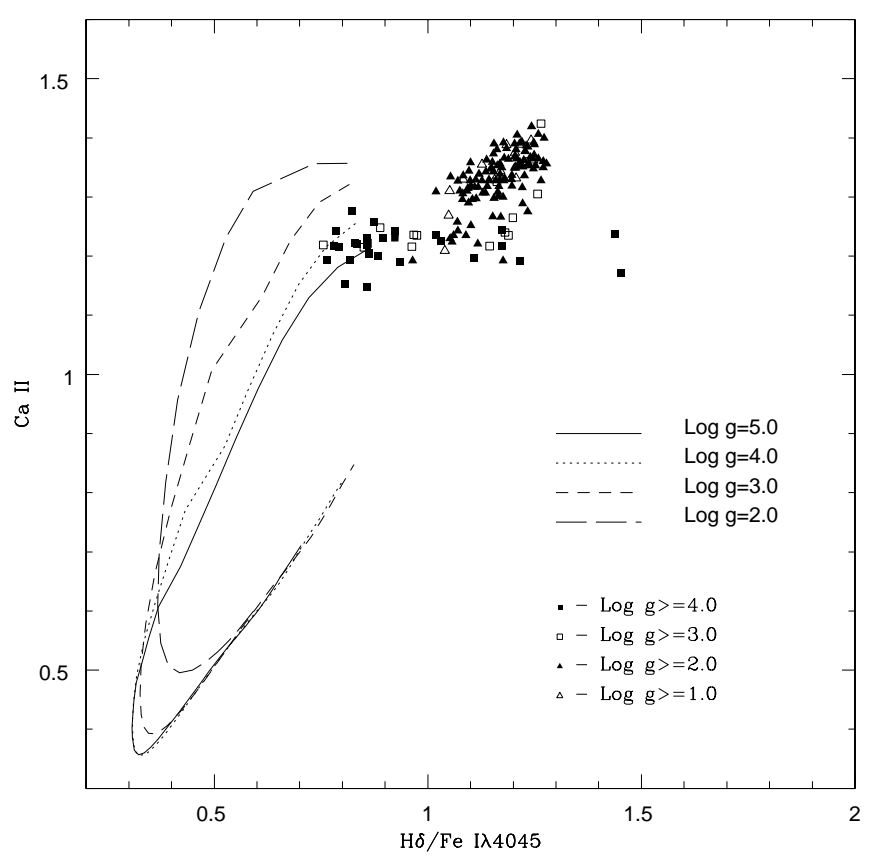

FIG. 8.-Same as Fig. 7, but with the synthetic curves truncated at an effective temperature of $6000 \mathrm{~K}$.

Figure 8. This is the same plot as Figure 7, but the synthetic curves have been truncated at $6000 \mathrm{~K}$ to illustrate where the empirical library takes over. The cutoff temperature was chosen to be well within the temperature range of the empirical fits to avoid any edge effects at the boundaries of the fits. A cutoff temperature of $5000 \mathrm{~K}$ was investigated to evaluate whether the increased continuity of the synthetic spectra would improve the results in the low-temperature region. The differences were not significant; hence, a cutoff of 6000 $\mathrm{K}$ was retained to maximize the use of empirical stars. At this temperature the metallicity sampling of the empirical library is good throughout the entire metallicity range. No steps were taken to induce continuity between the libraries at the interface.

\section{EVOLUTIONARY SYNTHESIS MODELS}

LR96 employed the evolutionary synthesis models of Bruzual \& Charlot 1993, hereafter BC93). Unfortunately, both the original BC93 spectral library and the higher resolution library of Jacoby, Hunter, \& Christian (1984), which replaced it in LR96, consist of solar metallicity stars only. Because chemical composition may have a significant impact on the derived age of a YSP, the present study utilizes the models of W94. In the W94 models, the isochrones of Bertelli et al. (1994; hereafter referred to as the Padua isochrones), which cover a range in metallicity, form the basis of the evolutionary synthesis approach.

The Worthey models employ empirical polynomial functions derived from fits to stellar index values for a library of stellar spectra. The fitting functions express how the stellar index strength varies as a function of effective temperature, gravity, and metallicity. From a Padua isochrone of a given age and metallicity and an assumed IMF, the models calculate the number of stars at each given point on the isochrone. Weighting the index value given by the fitting functions at that isochrone point by the luminosity and the number of stars for each point and then summing along the entire isochrone gives the index value for the total stellar population (Worthey \& Ottaviani 1997). See Charlot, Worthey, \& Bressan (1996) for how this methodology differs from other evolutionary synthesis models and the sources of discrepancies between different model sets.

\subsection{Resolution Matching}

Spectral broadening, due to both instrumental resolution and to stellar velocity broadening, redistributes the flux deficit out of the line center into the wings. Although the line ratio indices used here are fairly insensitive to spectral broadening, since both features that make up an index should be equally affected by broadening, it is still necessary to match the spectral resolutions of the model inputs and the observational data as closely as possible.

The broadening of the $\mathrm{MC}$ cluster spectra relative to the CFSL spectra used in the evolutionary synthesis models is determined as follows. First, since all MC cluster spectra are from a single observing run and all clusters have a similar low internal velocity dispersion, we use the very high $\mathrm{S} / \mathrm{N}$ integrated spectrum of the cluster 47 Tuc as our reference and compare it with the CFSL G2 stellar template star HD 10307. This latter template star is broadened with a variety of Gaussian dispersions and then cross-correlated against itself using the IRAF task FXCOR, and a fit is made to the width of the cross-correlation peak versus the $\sigma$ of the smoothing. Then, from a cross-correlation of the 47 Tuc spectrum with that of $\mathrm{HD} 10307$, it is determined that a broadening of $\sigma=1.17 \AA$ is required to match the CFSL spectra to that of the MC clusters. Similarly, it is determined that the spectra in the synthetic library need to be broadened by $\sigma=0.78 \AA$ to match the MC cluster spectra. Recalling that the MC cluster spectra are broadened with a $\sigma$ of $2.0 \AA$ before the spectral indices are measured, we apply the additional $2 \mathrm{~A}$ broadening to both the CFSL and SYNTHE spectra as well. The indices measured from clusters and stars can now be intercompared.

\subsection{Calculation of Integrated Line Features}

Since the diagnostic spectral indices involve quotients of the central intensities of pairs of absorption lines, taking linear combinations of the line ratios found in individual stars in building a model integrated stellar population will not produce correct results for the integrated indices. Instead, to model the integrated spectral indices, it is necessary to determine the individual residual central line intensities in the integrated model population before the final indices are formed. The W94 models use the input spectral libraries in the form of the fitting functions for the empirical data and an interpolation grid for the synthetic spectra. In each case the data used are the residual central line intensities of the library spectra, where the library spectra have been normalized to unity at $4000 \AA$. The central line intensities are measured by finding the minimum pixel value in a small range centered on the absorption line of interest. Spectral features $\mathrm{H} \delta, \mathrm{Ca}$ II $\mathrm{H}+\mathrm{H} \epsilon, \mathrm{Ca}$ II $\mathrm{K}$, and $\mathrm{Fe}$ I $\lambda 4045$ are measured for each star in the smoothed versions of the empirical and synthetic libraries. Polynomials are fitted to the individual empirical features, as discussed in $\S 4$ 4.1. In addition, for each synthetic spectrum, the average flux in the two wavebands for the BD index is calculated. To produce the integrated indices for a stellar population of a given age and metallicity, the W94 models calculate the integrated line features 
TABLE 6

Cluster Index Values

\begin{tabular}{|c|c|c|c|c|c|c|}
\hline Cluster & $\mathrm{H} \delta / \mathrm{Fe} \mathrm{I} \lambda 4045$ & $\pm 1 \sigma$ & Ca II & $\pm 1 \sigma$ & $\mathrm{BD}$ & $\pm 1 \sigma$ \\
\hline \multicolumn{7}{|c|}{ SMC } \\
\hline NGC $411 \ldots$ & 0.618 & 0.033 & 0.755 & 0.086 & 1.304 & 0.003 \\
\hline NGC $416 \ldots \ldots \ldots \ldots . . . .$. & 0.772 & 0.022 & 0.906 & 0.050 & 1.239 & 0.001 \\
\hline NGC 419 .................... & 0.607 & 0.015 & 0.673 & 0.030 & 1.329 & 0.001 \\
\hline \multicolumn{7}{|c|}{ LMC } \\
\hline NGC $1651 \ldots \ldots \ldots \ldots . . . .$. & 0.674 & 0.045 & 0.762 & 0.143 & 1.188 & 0.002 \\
\hline NGC $1751 \ldots \ldots \ldots \ldots . . .$. & 0.560 & 0.036 & 0.731 & 0.104 & 1.321 & 0.003 \\
\hline NGC $1754 \ldots$ & 0.747 & 0.020 & 0.782 & 0.037 & 1.312 & 0.001 \\
\hline NGC $1777 \ldots$. & 0.536 & 0.028 & 0.671 & 0.063 & 1.272 & 0.002 \\
\hline NGC $1783 \ldots \ldots \ldots \ldots . . .$. & 0.647 & 0.021 & 0.787 & 0.048 & 1.335 & 0.002 \\
\hline NGC $1795 \ldots \ldots \ldots \ldots . . . .$. & 0.672 & 0.047 & 0.802 & 0.130 & 1.248 & 0.003 \\
\hline NGC $1806 \ldots \ldots \ldots \ldots . . . .$. & 0.667 & 0.028 & 0.791 & 0.056 & 1.317 & 0.002 \\
\hline NGC $1818 \ldots \ldots \ldots \ldots . . .$. & 0.597 & 0.006 & 0.613 & 0.007 & 1.137 & 0.001 \\
\hline NGC $1831 \ldots \ldots \ldots \ldots . . . .$. & 0.462 & 0.008 & 0.493 & 0.012 & 1.435 & 0.001 \\
\hline NGC $1846 \ldots \ldots \ldots \ldots . . . .$. & 0.660 & 0.037 & 0.687 & 0.063 & 1.441 & 0.003 \\
\hline NGC $1866 \ldots \ldots \ldots \ldots . . .$. & 0.486 & 0.010 & 0.485 & 0.012 & 1.432 & 0.001 \\
\hline NGC $1868 \ldots \ldots \ldots \ldots . . . .$. & 0.521 & 0.015 & 0.663 & 0.035 & 1.428 & 0.001 \\
\hline NGC $1978 \ldots \ldots \ldots \ldots . . . .$. & 0.727 & 0.024 & 0.881 & 0.057 & 1.246 & 0.001 \\
\hline NGC $2010 \ldots \ldots \ldots \ldots . . .$. & 0.449 & 0.017 & 0.485 & 0.021 & 1.340 & 0.002 \\
\hline 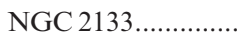 & 0.450 & 0.011 & 0.461 & 0.014 & 1.394 & 0.001 \\
\hline 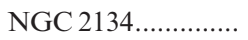 & 0.457 & 0.011 & 0.465 & 0.014 & 1.486 & 0.001 \\
\hline NGC $2136 \ldots \ldots \ldots \ldots . . . .$. & 0.499 & 0.008 & 0.539 & 0.011 & 1.352 & 0.001 \\
\hline NGC $2155 \ldots \ldots \ldots \ldots . . . .$. & 0.792 & 0.082 & 1.038 & 0.292 & 1.201 & 0.004 \\
\hline NGC $2162 \ldots \ldots \ldots \ldots$ & 0.620 & 0.038 & 0.700 & 0.073 & 1.337 & 0.003 \\
\hline NGC $2164 \ldots \ldots \ldots \ldots . . . .$. & 0.502 & 0.006 & 0.540 & 0.008 & 1.306 & 0.001 \\
\hline NGC $2173 \ldots \ldots \ldots \ldots . . . .$. & 0.691 & 0.033 & 0.749 & 0.072 & 1.326 & 0.002 \\
\hline NGC $2190 \ldots \ldots \ldots \ldots . . .$. & 0.596 & 0.053 & 0.655 & 0.090 & 1.315 & 0.003 \\
\hline NGC $2193 \ldots \ldots \ldots \ldots . . .$. & 0.668 & 0.040 & 0.860 & 0.097 & 1.299 & 0.003 \\
\hline NGC $2203 \ldots \ldots \ldots \ldots . . . .$. & 0.676 & 0.067 & 0.839 & 0.242 & 1.247 & 0.005 \\
\hline NGC $2210 \ldots \ldots \ldots \ldots . .$. & 0.707 & 0.016 & 0.799 & 0.031 & 1.310 & 0.001 \\
\hline NGC $2213 \ldots \ldots \ldots \ldots . . .$. & 0.657 & 0.021 & 0.745 & 0.042 & 1.425 & 0.002 \\
\hline 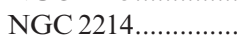 & 0.497 & 0.012 & 0.521 & 0.014 & 1.252 & 0.001 \\
\hline NGC $2249 \ldots \ldots \ldots \ldots$ & 0.477 & 0.018 & 0.556 & 0.036 & 1.317 & 0.002 \\
\hline \multicolumn{7}{|c|}{ Galactic Globulars } \\
\hline 47 Tuc ...................... & 0.900 & 0.009 & 1.239 & 0.028 & 1.231 & 0.000 \\
\hline M15 ......................... & 0.729 & 0.004 & 0.819 & 0.008 & 1.344 & 0.000 \\
\hline 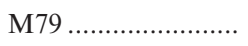 & 0.741 & 0.012 & 0.806 & 0.022 & 1.316 & 0.001 \\
\hline NGC $1851 \ldots \ldots \ldots \ldots . . . .$. & 0.788 & 0.006 & 0.968 & 0.016 & 1.310 & 0.000 \\
\hline
\end{tabular}

from the Padua isochrone of the desired age and $[\mathrm{Fe} / \mathrm{H}]$. The isochrones specify the number of stars with a given set of atmospheric parameters present in the theoretical HR diagram. Weighting by the number and luminosity of stars of each type, an integrated continuum for the population is developed. By also adding up the normalized line features weighted by the number of stars and applying the continuum, the total integrated line fluxes are calculated (see W94 for details).

The MC clusters are already integrated stellar systems; hence, their spectral indices are measured directly. The cluster indices, and their errors, are given in Table 6.

\section{APPLICATION OF AGE DETERMINATION METHOD}

\subsection{Integrated Spectral Indices for Simple Stellar Populations}

Utilizing the above modelling procedures, we form the integrated spectral indices for stellar populations covering a variety of age and metallicity. In this manner we can track the time evolution of the indices for different chemical compositions. The observed cluster indices can be compared with the model indices to determine the ages and metallicities of the MC star clusters from their integrated spectra. Those ages and metallicities can then be checked for consistency with the more primary ages and metallicities derived for the clusters from their CMDs and from spectroscopy of individual cluster stars. In this section we first present the evolution of spectral indices as a function of age and chemical composition in two diagnostic diagrams, then discuss how we determine the cluster ages from the diagrams, and finally compare the derived ages with those found in the literature.

To begin with, we plot the $\mathrm{Ca}$ II index against the $\mathrm{H} \delta$ / $\lambda 4045$ index as a function of age to form a trajectory in this two-index space. A trajectory is computed for each desired metallicity. By overplotting the observed MC cluster indices, the metallicity of a cluster can be determined from the relative location of the cluster point with respect to the 


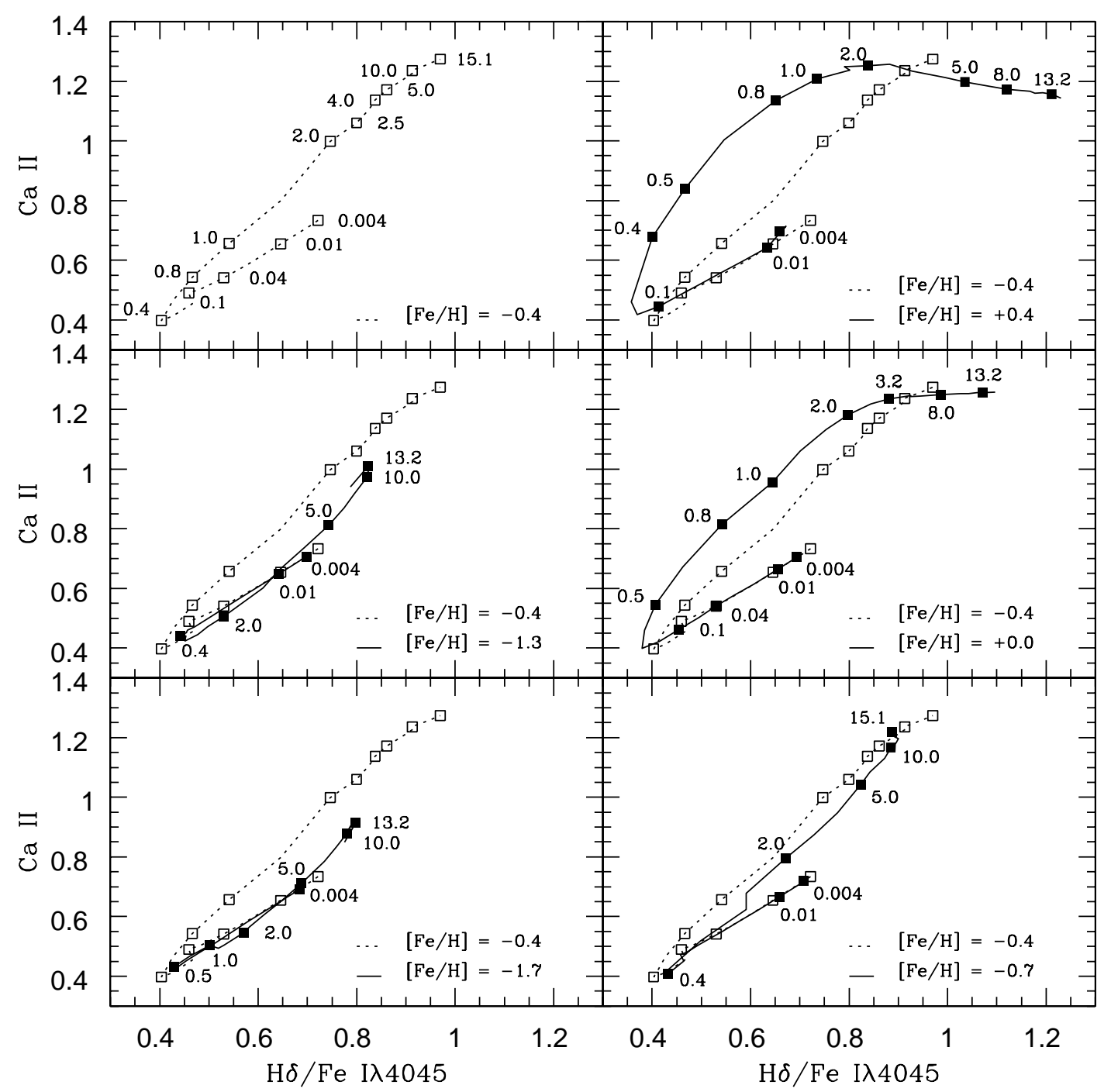

FIG. 9. - $\mathrm{H} \delta / \mathrm{Fe}$ I $\lambda 4045$ vs. Ca II as a function of age and metallicity. The top left panel shows the index evolution for [Fe $/ \mathrm{H}]=-0.4$. The open squares are the index values for the stellar population at a given age labelled in gigayears. This curve is reproduced as a dotted line in the other panels for comparison purposes. The other panels display (solid lines) the index trajectories for other metallicities as labelled in the figure.

trajectories, and its age determined by where it falls along a particular track. The process is repeated for the BD index resulting in two separate determinations. The trajectories are illustrated in Figure 9, where the $\mathrm{Ca}$ II index is plotted against the $\mathrm{H} \delta / \lambda 4045$ index for integrated stellar populations at six different metallicities. The indices were computed at the same spectral resolution as the observed MC cluster spectra following the prescription in $\S 5.1$. A similar plot for the BD index is given in Figure 10. The top left panel in each figure shows the evolution for $[\mathrm{Fe} / \mathrm{H}]=-0.4$, and this trajectory is plotted in all the succeeding panels for comparison purposes. The symbols represent the index values for an integrated stellar population of the given metallicity at different ages, marked in gigayears. The trajectories show the path that a cluster travels in the index space as it grows older. The need to model metallicity, which prompted the change to the W94 models from the BC93 models used in LR96, is apparent in the figures. Without an adequate account for metallicity, an old, metal-poor cluster could be mistaken for a significantly younger cluster at solar metallicity. Although the general behavior of the indices is very similar for different metallicities, the absorption feature index evolution is compressed as metallicity decreases. Since the BD index is relatively insensitive to metallicity, the compression is less drastic in the BD index tracks, with most of the effect arising from the $\mathrm{H} \delta / \lambda 4045$ index.

Some significant features of the figures should be noted. For the most metal-poor models, there is a degeneracy in the $\mathrm{Ca}$ II index values for different ages. It is particularly evident for the $[\mathrm{Fe} / \mathrm{H}]=-1.7$ trajectory in Figure 9. The youngest clusters (age $=0-0.4 \mathrm{Gyr}$ ) have the same $\mathrm{Ca}$ II and $\mathrm{H} \delta / \lambda 4045$ indices as those of the intermediate clusters (age $=0.5-5.0 \mathrm{Gyr}$ ). It is primarily this degeneracy that necessitates the inclusion of the $\mathrm{BD}$ index. As can be seen in Figure 10, the BD index significantly separates the youngest clusters from the intermediate-age clusters and enables simultaneous age and metallicity determination.

There is another degeneracy in the figures that affects the youngest clusters. The sections of the trajectories from an age of 0.004 Gyr to the "hook" at $\sim 0.4$ Gyr (for $[\mathrm{Fe} / \mathrm{H}]=-0.4)$ are superposed for all metallicities. This behavior occurs for both the $\mathrm{Ca}$ II index and the BD index, 


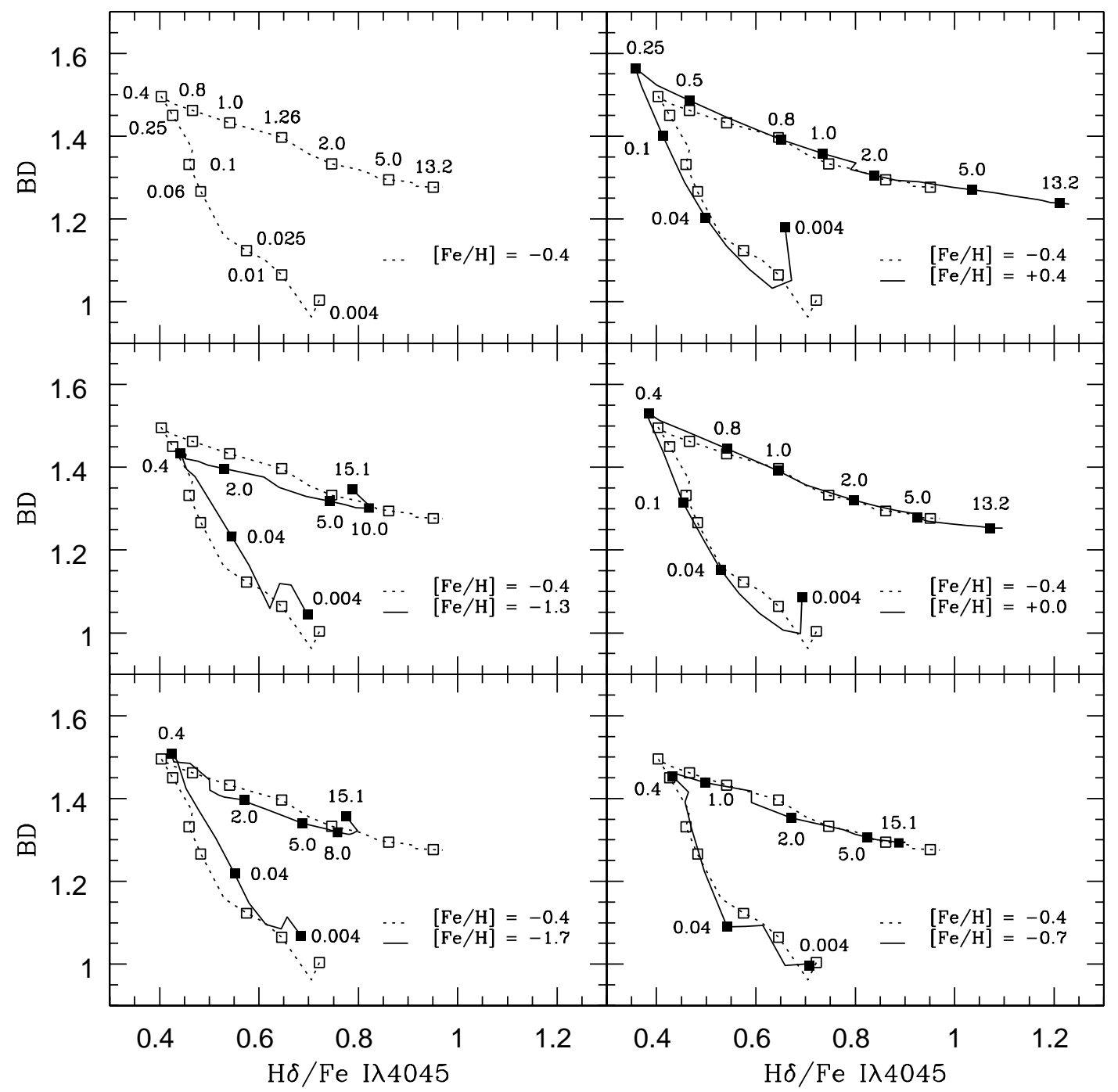

FIG. 10. - Same as Fig. 9, but with the Balmer discontinuity index plotted vs. H $\delta / \mathrm{Fe}$ I $\lambda 4045$

thereby rendering the determination of metallicities for these youngest populations essentially impossible. The derived age is also affected since the timescale from $0 \mathrm{Gyr}$ to the hook depends on the metallicity of the cluster.

The observed index values for the MC clusters listed in Table 6 are plotted on the model trajectories in Figures 1120 (note the expanded scales in Figs. 11, 12, and 16). The clusters are assigned to the figures based on their literature metallicities given in Table 2. Since no clusters have a derived $[\mathrm{Fe} / \mathrm{H}] \sim+0.4$, that plot was omitted. The error bars were calculated following the procedure described in $\S 3.4$. It is apparent in the figures that the clusters generally fall along the index tracks, especially in the $\mathrm{Ca}$ II versus $\mathrm{H} \delta$ / $\lambda 4045$ diagrams. This agreement provides an encouraging indication that the model trajectories are in accordance with reality. However, there are some clusters in the BD index plots that do not coincide with the model predictions. The most glaring examples are in Figures 19 and 20. As described in $\S 3.4$, spectrophotometric and/or background subtraction errors could be responsible for the $\sim 0.1$ discrepancy between modelled and observed BD indices. However, we note that the discrepancy appears to be correlated with metallicity, such that the more metal-rich clusters in general are the ones with the largest discrepancies between models and observations. Thus, we speculate that the problem is due to deficiencies in either the isochrones or the synthetic spectra for the more metal-rich systems.

\subsection{Age and Metallicity Determination}

Ages and metallicities for the MC clusters have been derived in the following manner. As is evident from the figures, model trajectories have been computed at six metallicities in both the $\mathrm{Ca}$ II versus $\mathrm{H} \delta / \lambda 4045$ and the $\mathrm{BD}$ versus $\mathrm{H} \delta / \lambda 4045$ diagrams. For a given metallicity trajectory in the $\mathrm{Ca}$ II diagram, at each computed model age point a test is made to see whether the observed indices for a particular cluster, when projected perpendicularly onto the model trajectory defined by the given age point and the next higher age point, lie between the two model ages. If so, a distance is computed for the line connecting the cluster indices and the projected position on the metallicity trajectory. Also, an age is interpolated based on the relative position of the projected cluster between the two model ages. For each metallicity trajectory, candidate ages and distances are calculated in this way. Then the minimum distance, as well as 


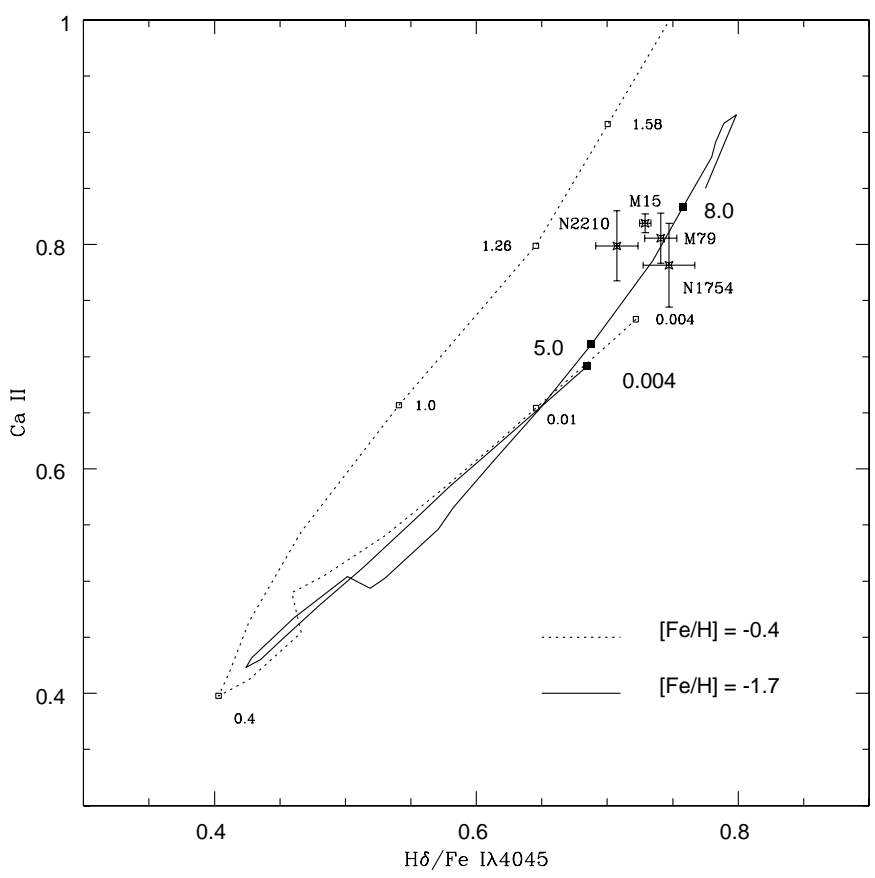

FIG. 11. $-\mathrm{H} \delta / \mathrm{Fe}$ I $\lambda 4045$ vs. $\mathrm{Ca}$ II for $[\mathrm{Fe} / \mathrm{H}]=-1.7$. The trajectories are the same as in Fig. 9, but now the MC cluster index data are included. Clusters are placed on this and the subsequent $\mathrm{Ca}$ II figures based on their literature metallicity values given in Table 2 . The dotted line is the trajectory for $[\mathrm{Fe} / \mathrm{H}]=-0.4$ with ages displayed as small labels, while the solid line is the track for $[\mathrm{Fe} / \mathrm{H}]=-1.7$ with large labels to mark the ages. All labelled ages are in gigayears. The two LMC clusters, NGC 2210 and NGC 1754 , occupy the same region of the diagram as the Galactic globular M79. These two clusters may have blue horizontal-branch stars contaminating their spectral indices (see text).

associated cluster age, is determined for each metallicity trajectory, thereby producing six candidate ages with their associated metallicity. A final age and metallicity for the cluster is computed by using a weighted average of three

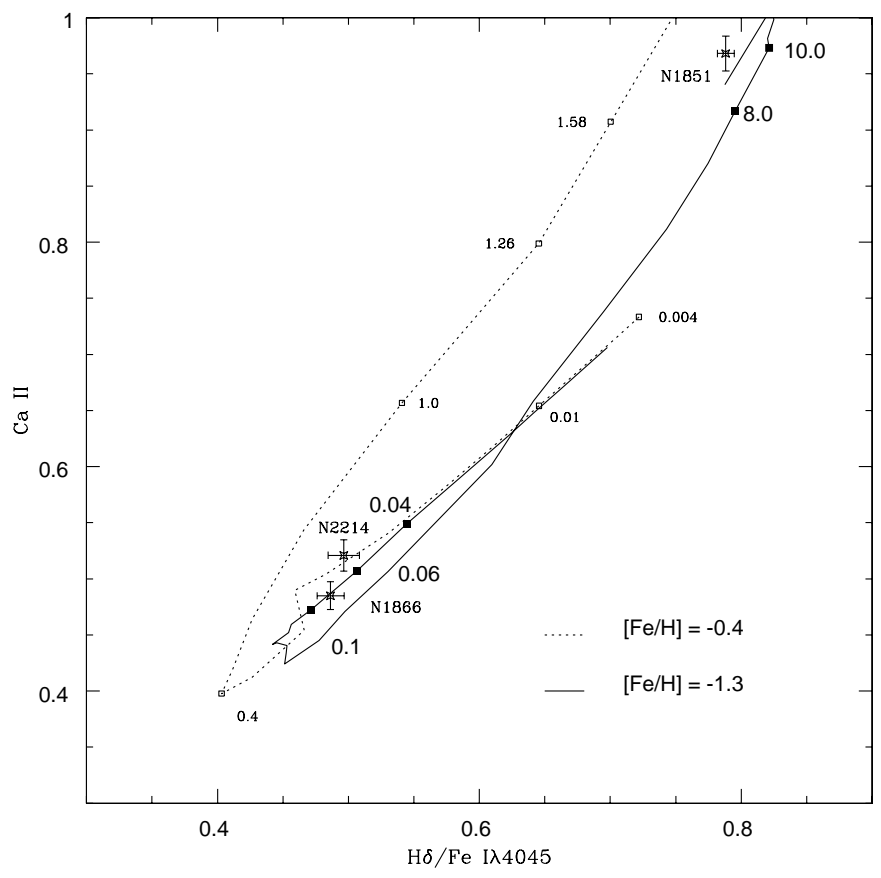

FIG. 12.-Same as Fig. 11, but with $[\mathrm{Fe} / \mathrm{H}]=-1.3$

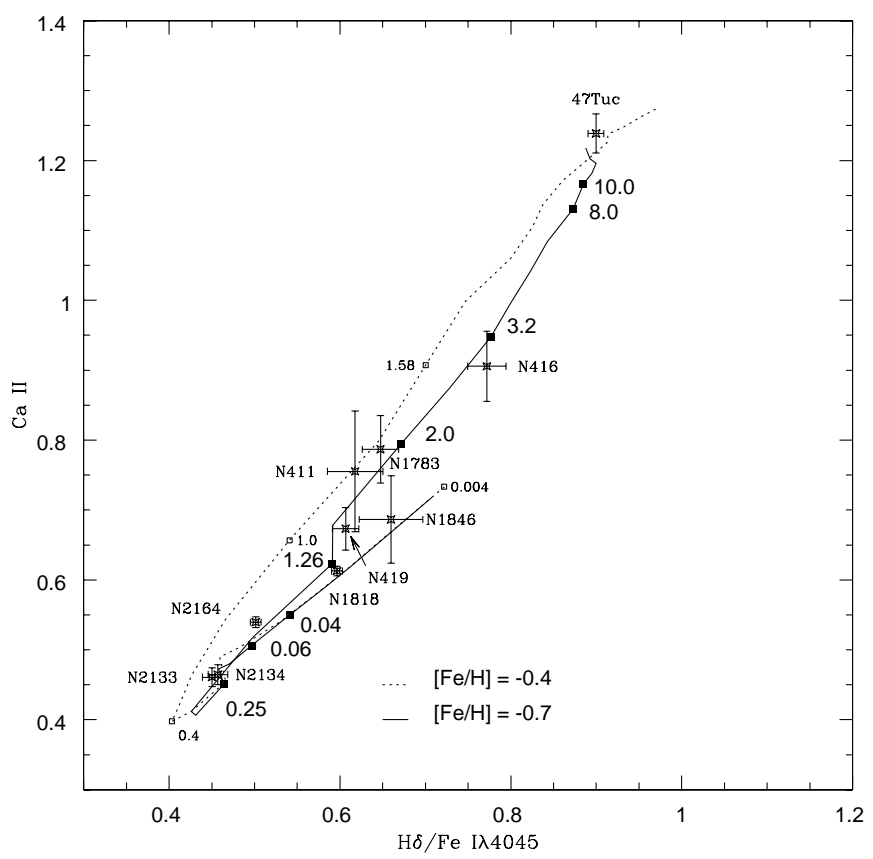

FIG. 13. - Same as Fig. 11, but with $[\mathrm{Fe} / \mathrm{H}]=-0.7$

values. These are the values associated with the minimum distance among the candidates and also the values associated with the next higher and lower metallicities. The weights used are simply the inverse of the three distances. If no candidate values have been found for a particular metallicity, then the next available metallicity is used.

The procedure used for computing ages and metallicities from the BD diagram is slightly different. Since the BD index provides virtually no metallicity information (see Fig. 10), we use the $\mathrm{Ca}$ II diagram results to constrain the final metallicity. For the BD results, we produce candidate ages and metallicities only at the two metallicity trajectories that

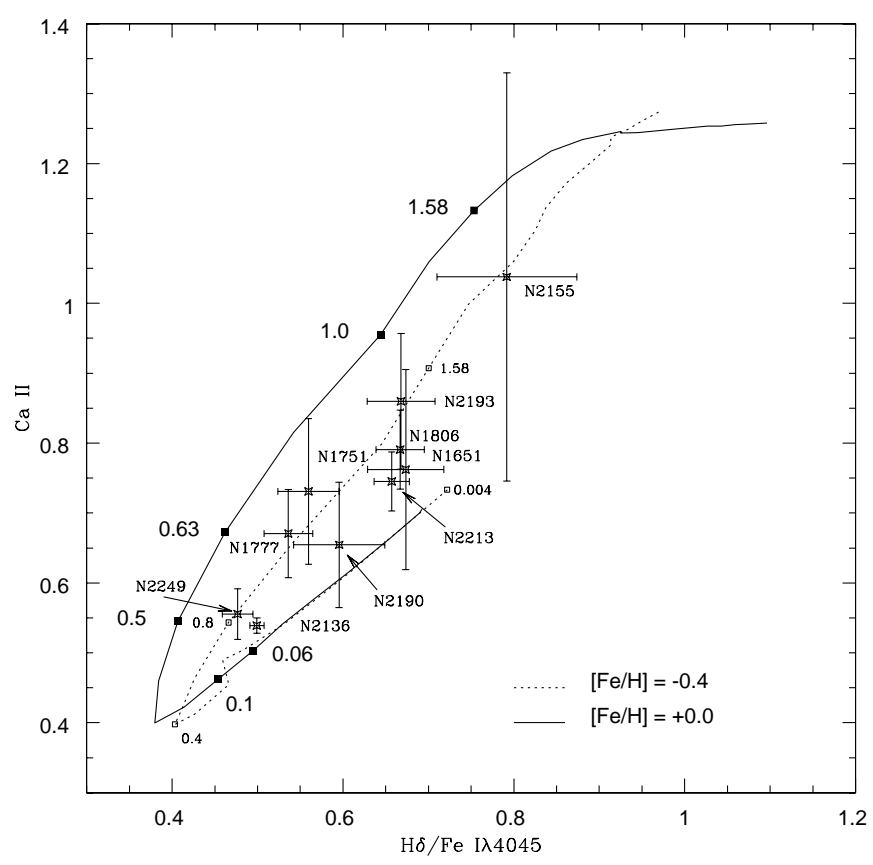

FIG. 14. - Same as Fig. 11, but with $[\mathrm{Fe} / \mathrm{H}]=+0.0$ 


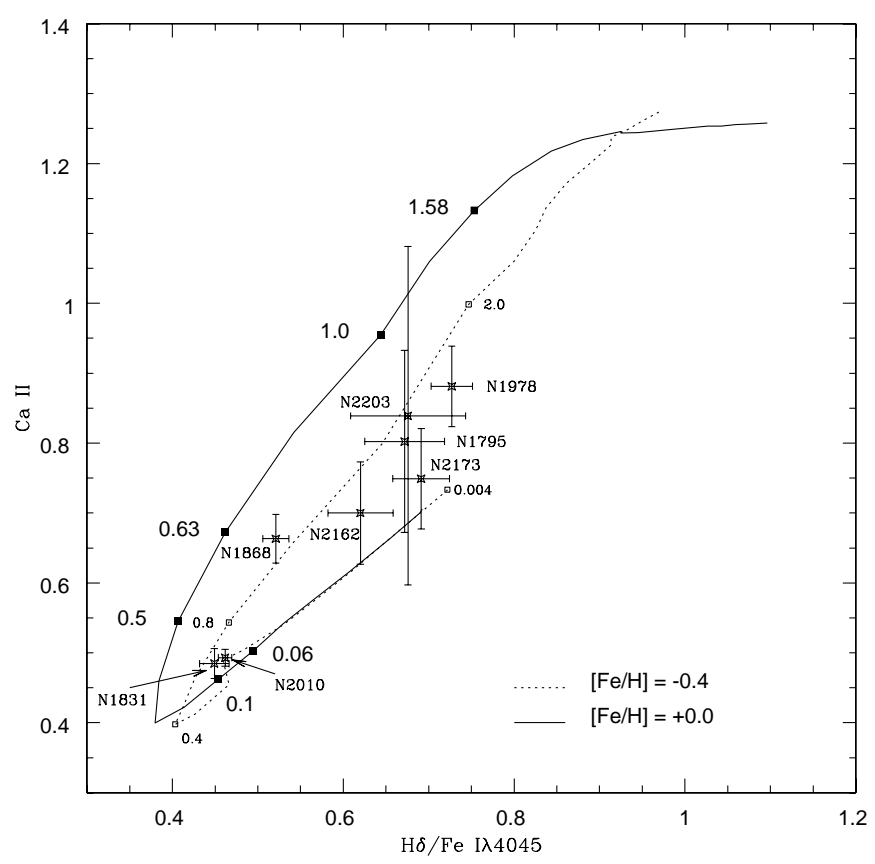

FIG. 15.- Same as Fig. 14

bracket the final metallicity found from the Ca II diagram. We then take a weighted average (by inverse distance) of the minimum distance ages and metallicities determined from the two bracketing metallicities. Thus, we emphasize that the $\mathrm{BD}$ metallicities are not independent of the $\mathrm{Ca}$ II metallicities.

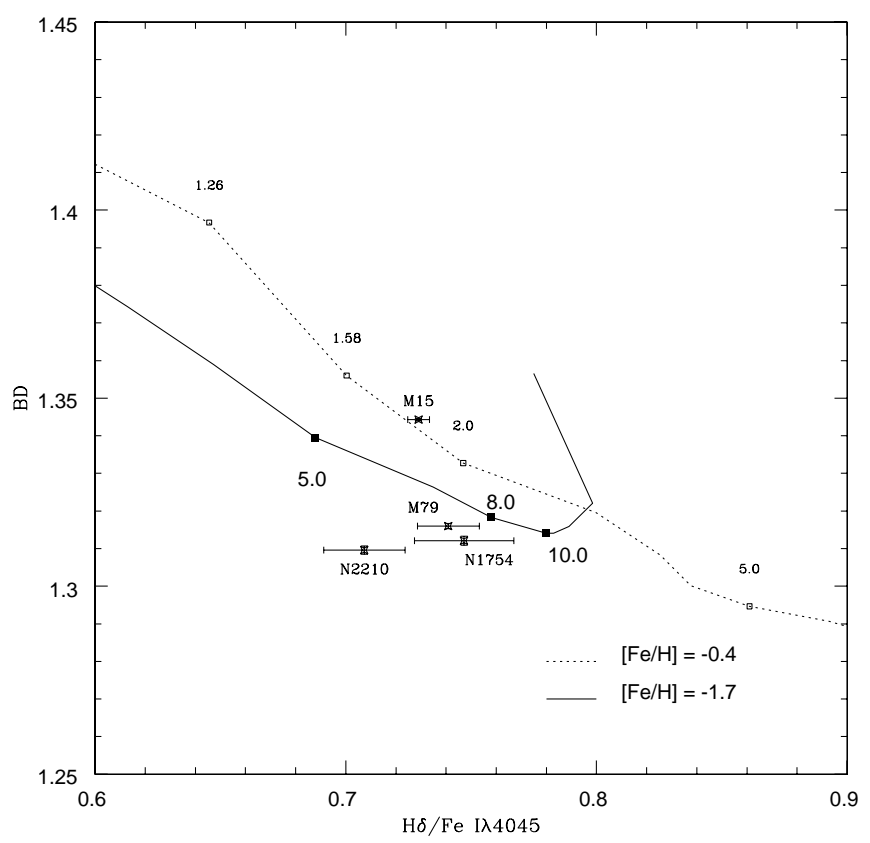

FIG. 16. - H $\delta / \mathrm{Fe}$ I $\lambda 4045$ vs. BD for $[\mathrm{Fe} / \mathrm{H}]=-1.7$. The trajectories are the same as in Fig. 10, but now the MC cluster index data are included. Clusters are placed on this and the subsequent $\mathrm{BD}$ figures based on their literature metallicity values given in Table 2 . The dotted line is the trajectory for $[\mathrm{Fe} / \mathrm{H}]=-0.4$ with ages displayed as small labels, while the solid line is the track for $[\mathrm{Fe} / \mathrm{H}]=-1.7$ with large labels to mark the ages. All labelled ages are in gigayears. Again, NGC 2210 and NGC 1754 occupy similar regions of the diagram as M15 and M79.

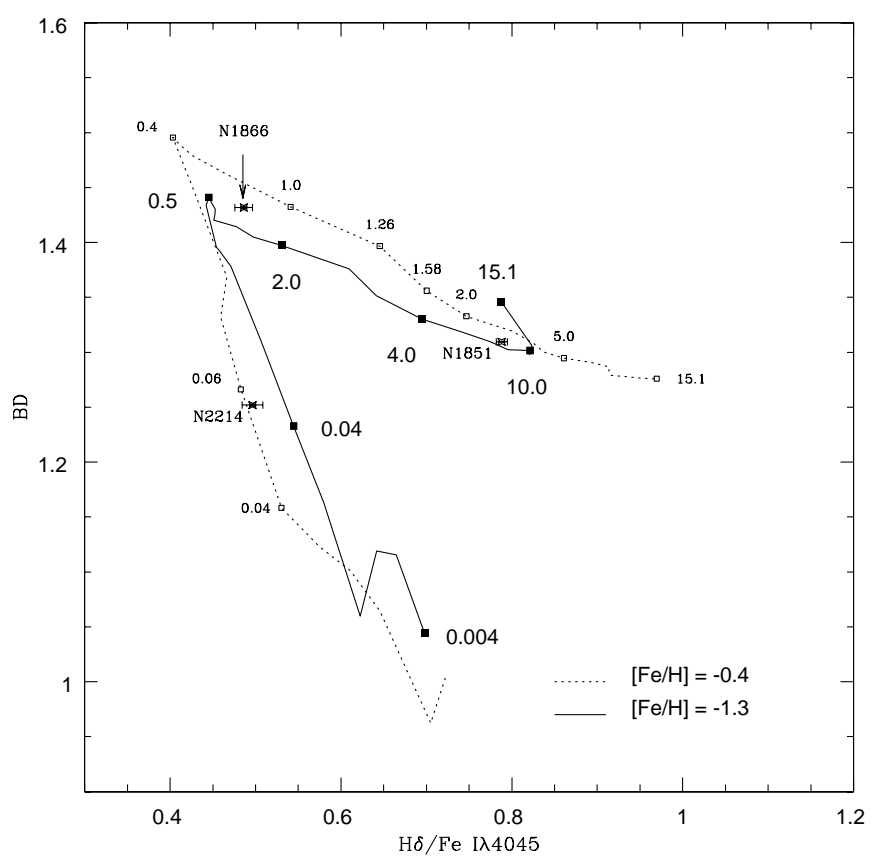

FIG. 17.-Same as Fig. 16, but with $[\mathrm{Fe} / \mathrm{H}]=-1.3$

As mentioned in the previous section, there can be a degeneracy in the age inferred from the $\mathrm{Ca}$ II diagram if a cluster is either very young or in the hook region of the diagram. If such a degeneracy is evident, then we use the location of the cluster in the $\mathrm{BD}$ diagram to determine whether to go with the younger, as opposed to the older, age possibility from the Ca II diagram. Likewise, if the cluster is either very young or in the hook region of the diagram, then no reliable metallicity information can be extracted. For instance, NGC 1818 in Figure 13 lies on the young age arm

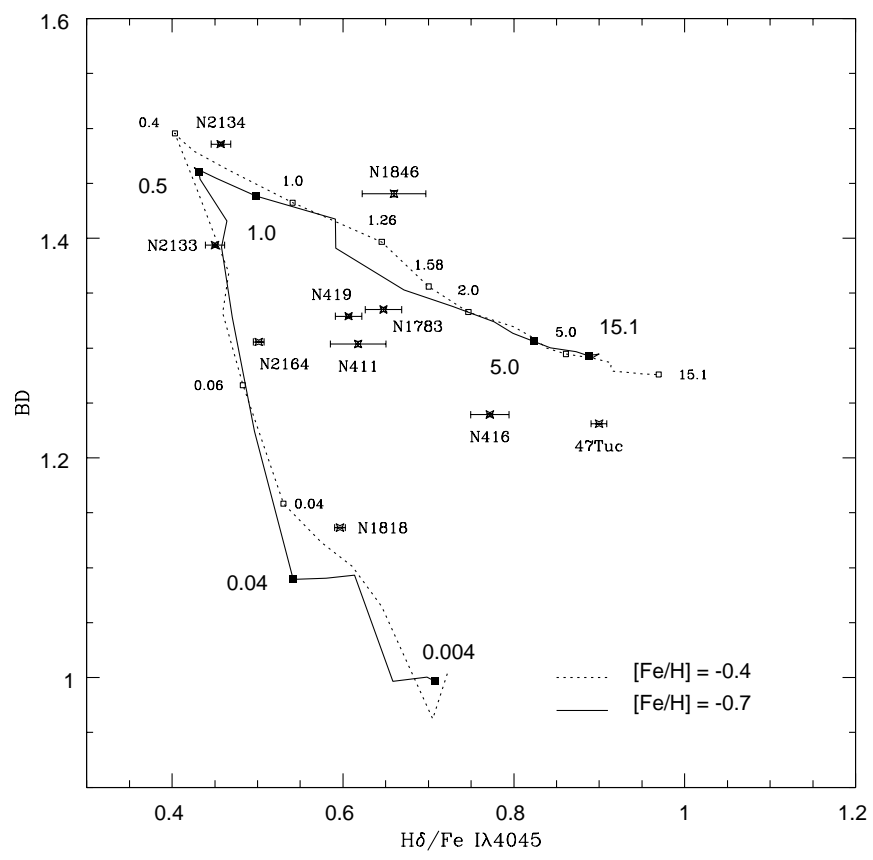

FIG. 18.-Same as Fig. 16, but with $[\mathrm{Fe} / \mathrm{H}]=-0.7$. The SMC clusters NGC 411 and NGC 416 both lie far enough from all the BD index tracks to make age determinations impossible for these clusters. 


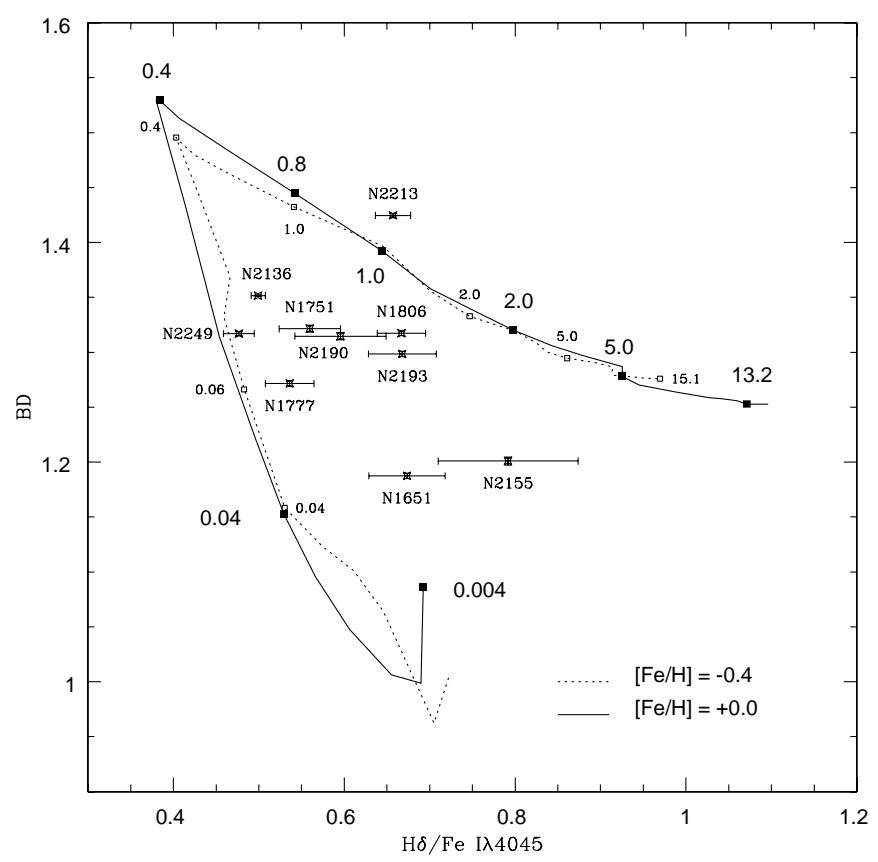

FIG. 19.-Same as Fig. 16, but with $[\mathrm{Fe} / \mathrm{H}]=+0.0$. Again, note here and in Fig. 20 the group of clusters whose BD index values prevent any age determinations because of their locations in the index space.

of the trajectory for all six metallicities. The BD index in Figure 18 provides no additional insight, since NGC 1818 lies in a similar position for all six BD trajectories. Fortunately, in these cases, the ages given by all the trajectories are quite close, with a slight trend to younger ages at higher metallicity, so that the derived age is not sensitive to the weighting of different metallicity trajectories in the final result.

For some clusters, even though a well-determined Ca II age can be obtained, deriving an age from the BD index is

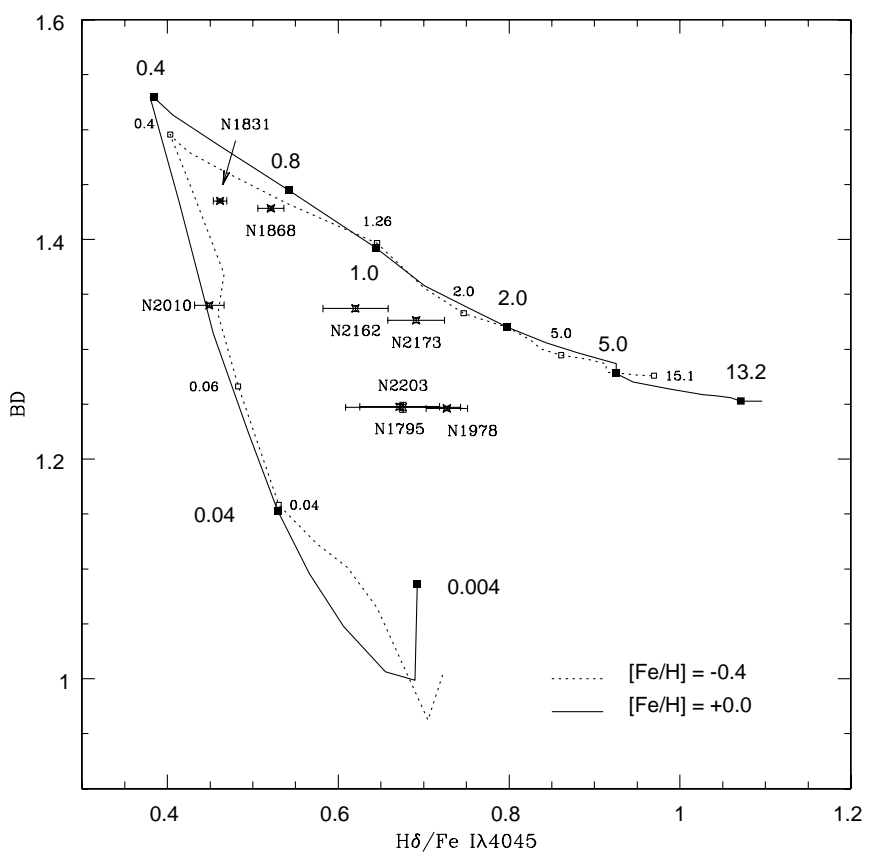

FIg. 20.- Same as Fig. 19 inconclusive. The measured value places the cluster in a region of the diagram that is not close to any of the model trajectories. As is discussed in $\S \S 3.4$ and 6.1, it is unclear whether the discrepancy between models and data is due to errors in the observations or in the models or in both.

Errors have been determined in the ages derived from the $\mathrm{Ca}$ II diagram and the $\mathrm{BD}$ diagram, as well as in the derived metallicities, in the following manner. We computed revised $\mathrm{Ca} \mathrm{II}, \mathrm{BD}$, and $\mathrm{H} \delta / \lambda 4045$ indices by adding the $1 \sigma$ index errors to the original indices and computed another index set by subtracting the $1 \sigma$ index errors. For these revised indices we utilized the same age and metallicity determination procedure as described above to derive ages and metallicities. We then used the mean absolute difference between the original age and these revised ages as a measure of the $\pm 1 \sigma$ error in the age, and similarly for the metallicities. Because the uncertainties in age determinations are more symmetric when considered in log age, we present all our age results in log format.

The derived ages from the $\mathrm{Ca}$ II indices, and their $\pm 1 \sigma$ errors, are given in columns (2) and (3) of Table 7, while the derived ages and errors from the BD indices are given in columns (4) and (5). Final ages are assigned to the cluster by averaging the individual determinations of the $\mathrm{Ca}$ II and BD indices. For the clusters whose BD index is inconclusive, the $\mathrm{Ca}$ II age is assigned as the final age. Though the Ca II ages are more reliable as a result of the metallicity discrimination inherent in the diagrams, no specific weightings are given to one index or the other. It will be seen that this is a moot issue for a majority of the clusters because the two determinations are very close in most cases. The final derived ages are given in column (6) of Table 7 and the associated $1 \sigma$ errors are in column (7). Similarly, the $[\mathrm{Fe} / \mathrm{H}]$ values derived from the $\mathrm{Ca}$ II diagram and associated $\pm 1 \sigma$ errors are listed in columns (8) and (9), while those determined from the BD diagram are given in columns (10) and (11). Final values for $[\mathrm{Fe} / \mathrm{H}]$, taken from a straight average of those determined from the $\mathrm{Ca}$ II and BD methods, are listed in column (12). Note that the errors given in column (13) are identical to those from the $\mathrm{Ca}$ II index determinations. The $[\mathrm{Fe} / \mathrm{H}]$ values extracted from the BD diagram are sufficiently coupled to those obtained from the $\mathrm{Ca}$ II diagram that we do not consider the errors to be lowered by averaging the two results. In the next section various clusters are discussed on an individual basis.

\subsection{Notes on Individual Clusters}

The following clusters have an observed BD index that does not allow a conclusive age determination: NGC 1651, 1751, 1777, 1795, 1978, 2155, 2193, 2203, 411, and 416.

The following clusters are in the hook region for the $\mathrm{Ca}$ II index, but the $\mathrm{BD}$ index clearly selects a young age so the young solution for $\mathrm{Ca}$ II is chosen. No metallicity determination is possible: NGC 2010, 2133, 2164, 2214.

NGC 1754 and NGC 2210: According to the literature these two clusters are of Galactic globular cluster age and very metal-poor. The $\sim 4-6$ Gyr ages derived from our models are most likely due to the contamination of the indices by a blue horizontal branch. The W94 models only treat the horizontal branch as a red clump (Leonardi \& Worthey 2000). A blue horizontal branch will decrease the value of the Ca II index, causing the clusters to appear to have a 
TABLE 7

Derived MC Cluster Ages

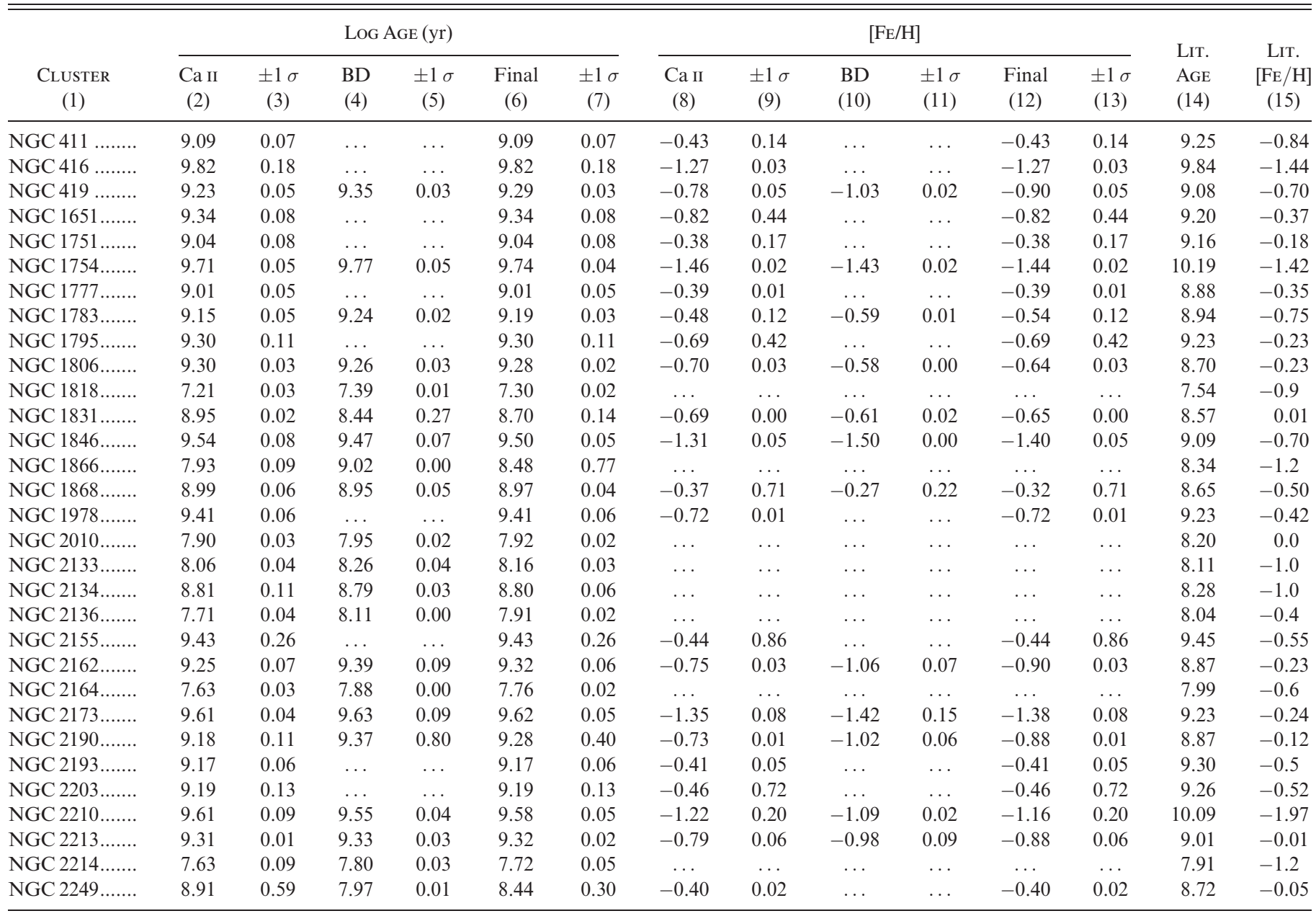

younger age. As can be seen in Figure 11, these two clusters reside in the same region of the diagram as M15, a very old, metal-poor Galactic globular cluster with a blue horizontal branch.

NGC 1818: This cluster is very young so no metallicity determinations can be made.

NGC 1831: This cluster potentially has another set of determinations. It is situated in the hook region of both the $\mathrm{Ca}$ II and the BD diagrams, so it has both a younger solution (age $=0.19 \mathrm{Gyr}$ and $[\mathrm{Fe} / \mathrm{H}]=-0.50)$ and an older solution. The older solution is selected because it has more selfconsistency between the $\mathrm{Ca}$ II and $\mathrm{BD}$ age derivations.

NGC 1846: Determinations from the BD index are uncertain because the index point is significantly above all the metallicity trajectories.

NGC 1866: The Ca II (0.09 Gyr) and BD (1.0 Gyr) ages are in serious disagreement.

NGC 2134: The Ca II index places the cluster in the hook region, but the $\mathrm{BD}$ index clearly selects an older solution.

NGC 2136: The $\mathrm{Ca}$ II index has multiple solutions, but the $\mathrm{BD}$ index indicates a young age, so the younger solution is selected. No metallicity determination is possible.

NGC 2190: The BD index determinations are somewhat uncertain because the index point falls in between all the trajectories, making interpolation difficult.

NGC 2249: The age determinations for the BD index $(0.08 \mathrm{Gyr})$ and the $\mathrm{Ca}$ II index $(0.8 \mathrm{Gyr})$ are in serious disagreement. The $\mathrm{Ca}$ II age is closer to the literature value of $0.5 \mathrm{Gyr}$, as given in Girardi et al. (1995).

\subsection{Reliability of Age and Metallicity Determinations}

To check the self-consistency of the age determinations, a least-squares fit was calculated for the derived $\mathrm{Ca}$ II ages and the BD ages. The best-fit line is displayed in Figure 21 for the clusters with age determinations for both indices. The agreement is excellent, with only NGC 1846 and NGC 2249 showing serious deviations. The calculated slope of the least-squares fit to the data is $0.82 \pm 0.10$, with an $r$-value of 0.89 . If we make a least-squares fit in linear age, rather than in the $\log$ of the age, the resulting slope is $1.01 \pm 0.07$, with an $r$-value of 0.96 . Thus, the two spectral indices produce nearly the same derived age for most of the clusters.

If we similarly compare the final age determinations with literature values, the agreement is still encouraging. In comparing our ages with literature data, we exclude the two oldest clusters, NGC 1754 and NGC 2210, which have the aforementioned problem that our derived ages are too young because of the lack of a blue HB in our models. The fit is shown in Figure 22. The correlation coefficient for the fit is $r=0.90$, while the slope is $0.85 \pm 0.08$, in the sense that our modelled ages show a larger age range than those from the literature. If we make the comparison in age, rather than in the $\log$, we find a slope of 0.86 , with an $r$-value of 0.83 . 


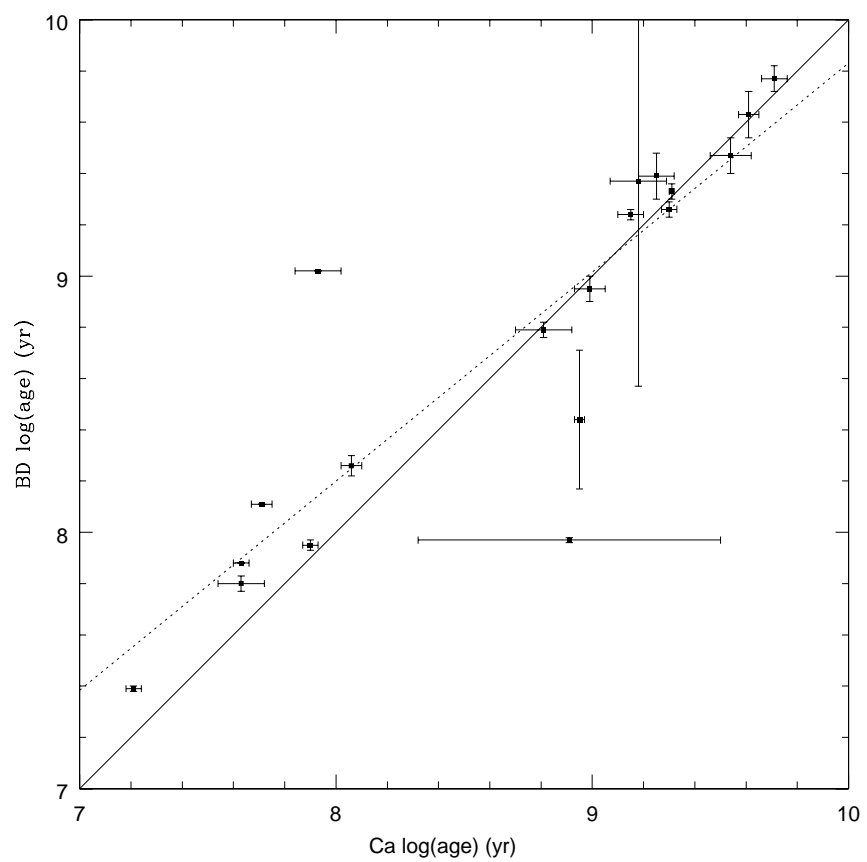

FIG. 21.-MC cluster ages derived from the $\mathrm{Ca}$ II index and the BD index are plotted against each other (filled squares) for the clusters which have determinations from both indices. The dotted line is a least-squares fit to the data, while the solid line denotes the identity relation.

We have also plotted (asterisks) age data for the clusters that we have in common with the Beasley et al. (2002) integrated-light study. The Beasley et al. (2002) ages are the average of the two values for each cluster given in their Table B1. While there are five clusters in common between our two studies, we exclude the results for NGC 1754, as mentioned above.

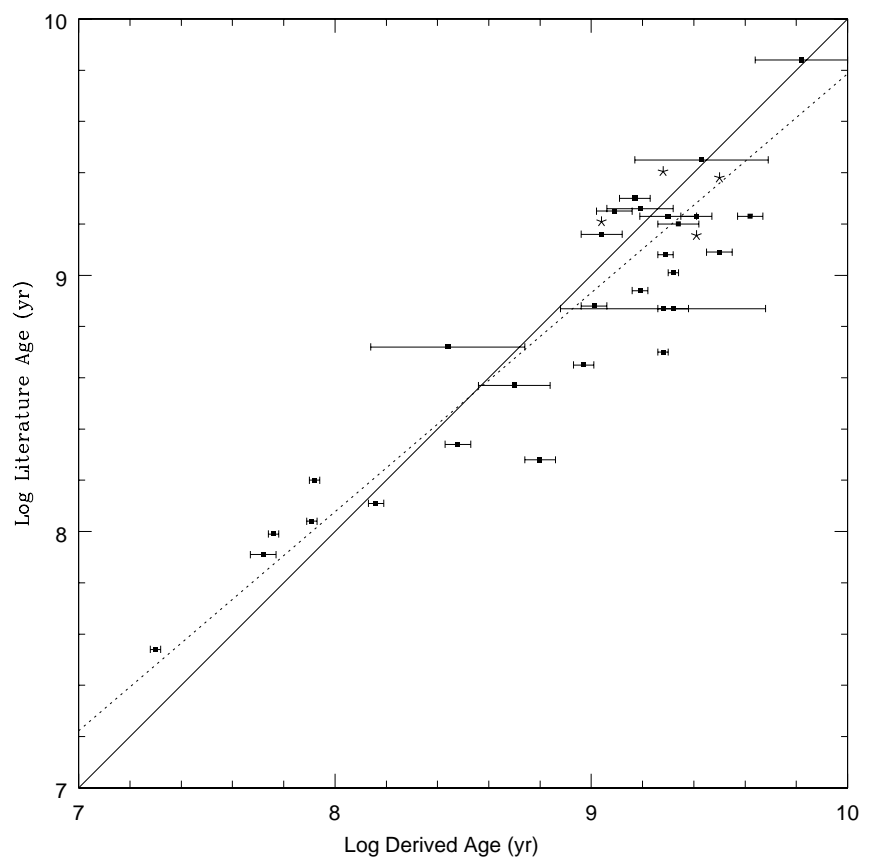

FIG. 22.-Final derived ages for the MC clusters are plotted vs. the literature values from Table 2 (filled squares). The dashed line is the best-fit line to the data, while the solid line denotes the identity relation. The asterisks show age determinations in common with the Beasley et al. (2002) results.

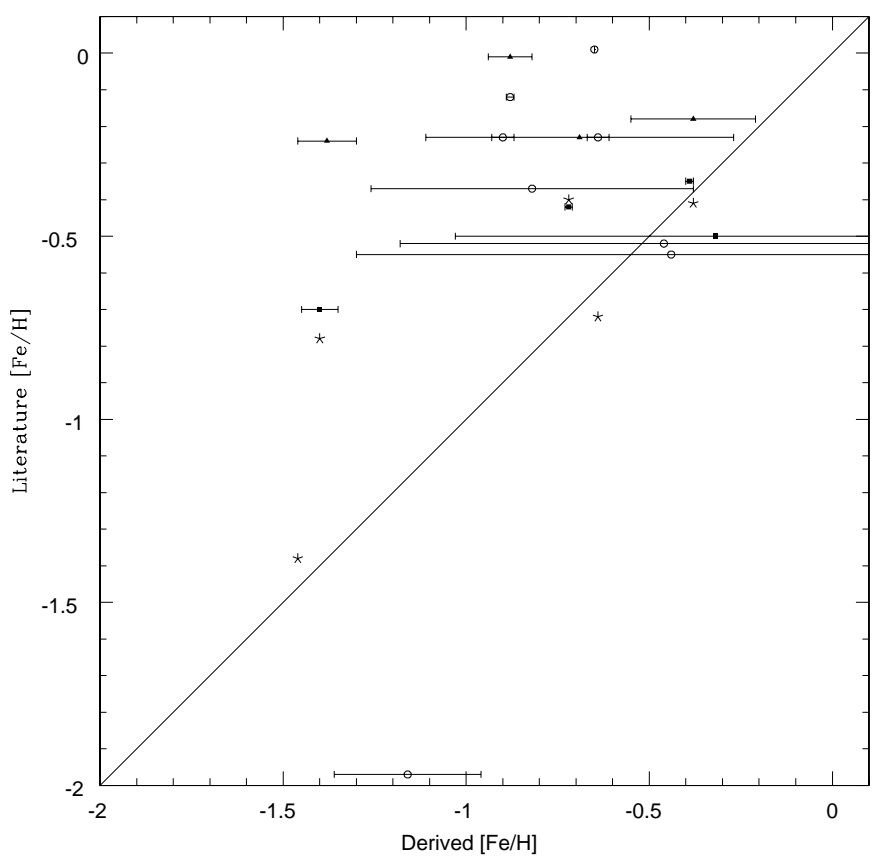

FIG. 23.-Final derived metallicities for the MC clusters are plotted vs. the Olszewski et al. (1991) values from Table 2. The data are divided into three age bins. Specifically, the open circles represent the youngest clusters with ages less than 9.1 in the log. The filled squares represent clusters with ages between 9.1 and 9.3 in the log, and the filled triangles are for the oldest clusters with ages (in gigayears) greater than 9.3 in the log. The solid line shows the identity line. The derived metallicities are systematically more metal-poor than the Olszewski et al. (1991) metallicities. Asterisks denote metallicity determinations in common with the Beasley et al. (2002) results.

While the agreement between our age determinations and those from the literature is encouraging, the comparison of our $[\mathrm{Fe} / \mathrm{H}]$ determinations with those from the literature, plotted in Figure 23, is less satisfactory. For the sake of homogeneity, we have only taken literature $[\mathrm{Fe} / \mathrm{H}]$ values from the work of Olszewski et al. (1991) and also plotted (asterisks) results for the five clusters in common between us and Beasley et al. (2002). While overall there is some trend between our values and those in Olszewski et al. (1991), there is a large scatter present, as well as a systematic offset at higher $[\mathrm{Fe} / \mathrm{H}]$. The large scatter $(\sim \pm 0.3 \mathrm{rms})$ seen at $[\mathrm{Fe} / \mathrm{H}]>-0.7$ (according to the Olszewski et al. 1991 values) is actually quite consistent with the fact that our methods for extracting age and metallicity from the integrated spectra are more sensitive to age than to metallicity. However, the offset between our $[\mathrm{Fe} / \mathrm{H}]$ values and those of Olszewski et al. (1991) at high $[\mathrm{Fe} / \mathrm{H}]$ is more troublesome. Restricting the data to only those clusters with Olszewski et al. (1991) $[\mathrm{Fe} / \mathrm{H}]$ values higher than -0.7 , we find a mean offset of $\Delta[\mathrm{Fe} / \mathrm{H}]=-0.42$, in the sense that our values are systematically lower than those of Olszewski et al. (1991). As can be seen in Figure 23, there is a suggestion that the $[\mathrm{Fe} / \mathrm{H}]$ offset between our results and those of Olszewski et al. (1991) increases with decreasing cluster age, in that the metallicity offset for the younger clusters (open circles) appears to be less than for the older clusters (filled triangles). However, the result is not definitive. No offset appears to be present between our metallicities and those from Beasley et al. (2002), which are plotted as asterisks.

Given the more fundamental nature of the $[\mathrm{Fe} / \mathrm{H}]$ determinations carried out by Olszewski et al. (1991), 
viz., Ca II triplet spectroscopy of individual giants, it is most likely that our abundances are systematically in error. On the other hand, Bica et al. (1998) noted a discrepancy of $\Delta[\mathrm{Fe} / \mathrm{H}] \sim-0.3$ between their $\mathrm{MC}$ cluster metallicities (derived from the GB in their CMDs) with the work of Olszewski et al. (1991); i.e., their discrepancy is of the same magnitude and direction as ours. Moreover, Geisler et al. (2003) find a metallicity offset with respect to Olszewski et al. (1991) in the same direction as ours, but with only about half the size. The Geisler et al. (2003) studies, as in the case of Bica et al. (1998), come from characteristics of the CMDs (in the Washington photometric system). In addition, while the number statistics are small, our results show no systematic offset with respect to Beasley et al. (2002). Thus, while the likelihood remains that our abundances need revision, a zero-point problem may be present as well in the Olszewski et al. (1991) abundance scale.

Returning to the comparison between our ages and literature ages in Figure 22 and to the fact that the slope of the relation between literature ages versus our ages is significantly less than unity (0.85), it is evident that our ages show a greater spread than the literature ages. The most likely explanation is that observational errors in the cluster spectral indices give rise to correlated errors in age and metallicity. Specifically, upon examining the location of NGC 1783 in Figure 13, it is evident that, if we assume a fixed metallicity for the cluster, e.g., $[\mathrm{Fe} / \mathrm{H}]=-0.4$, then the $\pm 1 \sigma$ observational errors will tend to affect the derived age by $\sim \pm 0.1 \mathrm{Gyr}$, while for an assumed fixed age, the $\pm 1 \sigma$ errors will affect the derived $[\mathrm{Fe} / \mathrm{H}]$ by $\sim \pm 0.4$. The problem is that an error in the $[\mathrm{Fe} / \mathrm{H}]$ determination of 0.4 will lead to a correlated error in the age determination of $\sim 0.6 \mathrm{Gyr}$, as a result of the slower evolution of the spectral indices for a more metal-poor population. Naturally, the specifics of the systematic age error associated with an error in $[\mathrm{Fe} / \mathrm{H}]$ is dependent on both the age and $[\mathrm{Fe} / \mathrm{H}]$ of a cluster, but the effect always works in the direction that an error in $[\mathrm{Fe} / \mathrm{H}]$, in the sense that $[\mathrm{Fe} / \mathrm{H}]$ is underestimated, leads to an associated overestimate of the age.

The previous discussion should clarify why our age determinations for the MC clusters spread to older ages than the literature values. The reasons are twofold. First, assuming that our $[\mathrm{Fe} / \mathrm{H}]$ determinations are systematically too metal-poor by -0.4 in $[\mathrm{Fe} / \mathrm{H}]$, the implication is that our ages are accordingly overestimated, which will draw out the higher end of the age distribution. Second, random errors in $[\mathrm{Fe} / \mathrm{H}]$ will create a corresponding scatter in age that will broaden out the age distribution and create an artificial agemetallicity relation (AMR) for the MC clusters. This latter problem has played a role in the long-standing controversy over the reality of an AMR in the Galactic disk (McClure \& Tinsley 1976; Feltzing, Holmberg, \& Hurley 2001 and references therein).

Based on the above discussion, we arrive at two principal conclusions:

1. The ages determined from direct modelling of the spectral indices of young stellar populations in integrated light are overall in good accord with those determined from the more fundamental analyses of CMDs.

2. The main impediment to obtaining high accuracy in age determinations is the difficulty in independently determining the metallicity from the integrated spectrum. Thus, the age-metallicity degeneracy that afflicts the reliable extraction of ages for older stellar populations is a significant factor for young populations as well.

\section{HISTORY OF CLUSTER FORMATION IN THE MAGELLANIC CLOUDS}

The integrated-light age determination method described here can be a powerful tool for examining the cluster formation history of the LMC, since the ages are all derived from a single method and from integrated spectra of fairly uniform quality. By simultaneously determining cluster ages and metallicities, the distribution of clusters in time and chemical composition can be determined, thus revealing the complete history of star formation and chemical enrichment. Having verified the basic reliability of the age/metallicity determinations from our integrated spectra, we now look at the distribution in age and metallicity of these clusters, with a particular view toward assessing the time dependence of the cluster formation rate.

It is known from a variety of studies that the star formation rate in the LMC has not been constant over its lifetime. From age data based on compilations of cluster CMDs, it is now generally agreed that a pronounced gap appears in the LMC cluster age distribution from $\sim 3$ to $\sim 12$ Gyr (Jensen, Mould, \& Reid 1988; Da Costa 1991; van den Bergh 1991; Geisler et al. 1997; Rich, Shara, \& Zurek 2001; Piatti et al. 2002). A second, smaller minimum in the cluster formation rate appears to be present from $0.2-0.7 \mathrm{Gyr}$, or $\log t=8.3$ 8.8 (Barbero et al. 1990; van den Bergh 1991). While the cluster age distribution determined from integrated $U B V$ colors initially did not reveal age gaps or peaks, a subsequent reevaluation, incorporating both stochastic effects and metallicity effects on the integrated colors, does indeed produce similar age gaps as for the CMD-based age distributions (Girardi et al. 1995). In addition, the derived age gap between 3 and 12 Gyr appears to be accompanied by a metallicity gap between $[\mathrm{Fe} / \mathrm{H}]=-0.7$ and $[\mathrm{Fe} / \mathrm{H}]=-1.5$ (Olszewski et al. 1991). In contrast, the cluster formation history of the SMC appears to be quite different from and, in fact, complementary to that of the LMC, in that very few truly old SMC clusters are found, while several are found within the large LMC age gap (Da Costa 1991; Piatti et al. 2002). Studies of the star formation history in the LMC field stars are generally in accord with the cluster age distribution, in that the star formation rate appears to have gone through a major upswing starting about 3 Gyr ago. However, the deep lull between 3 and 12 Gyr found in the cluster formation rate is not as evident in the field star formation rate (Butcher 1977; Geha et al. 1999; Olsen 1999; Holtzman et al. 1999; Harris \& Zaritsky 2001). Thus, on the whole the latter studies find some decoupling between field and cluster star formation and chemical enrichment histories.

Unfortunately, the age determination method used in this paper is not well suited to explore the intermediate age gap. The spectral indices employed here are optimized to distinguish the younger ages, and they lose their discriminating power for ages greater than $\sim 4$ Gyr. Moreover, the MC clusters studied were selected for their younger ages and thus do not represent an unbiased sample of MC clusters. However, we can examine the age distribution of our sample of clusters for nonuniformity in the cluster formation rate over time and compare that with the literature data. Hence, 

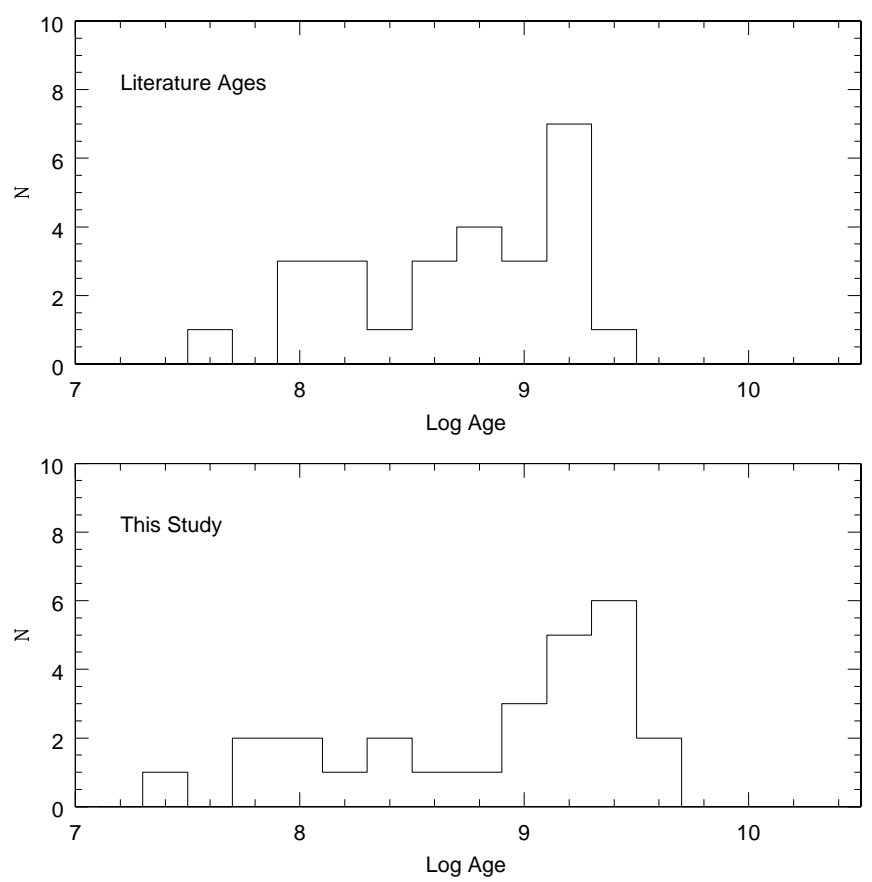

FIG. 24.- Histogram comparison of the derived LMC cluster age distribution (bottom) vs. their literature ages (top). We have excluded the two clusters (NGC 1754 and NGC 2210) with a literature age $\sim 15$ Gyr.

in Figure 24 we plot a histogram of the LMC cluster ages derived in this study, as well as one derived from literature values. The width of the bins is 0.2 in $\log t$ in gigayears, and again we have excluded the two oldest clusters (NGC 1754 and NGC 2210) from the analysis for which we derive discrepant ages, because of the lack of blue HB stars in the population models. The peak in the age distribution derived from the spectral indices is offset by about one age bin from the literature distribution. As mentioned above, this shift probably arises from the systematically lower metallicities that we derive for the clusters, which then cause correspondingly older ages to be determined. Otherwise, the two distributions agree well. In particular, the sharp cutoff in the histogram of the literature-derived cluster ages at $\sim 3 \mathrm{Gyr}$ $(\log t=9.5)$ is also seen in the age distribution derived from our integrated light methods. In addition, given the logarithmic stretching of the age scale at younger ages in the histogram, it appears that cluster formation has been more concentrated at $\log t \sim 8.0$ than at $\log t \sim 8.5$. Specifically, a pronounced depletion in clusters between 0.2 and $0.7 \mathrm{Gyr}$ in age is readily seen when the clusters are plotted in linear age. In fact, the two clusters with ages near $\log t=8.5$ represent cases for which the $\mathrm{Ca}$ II ages and $\mathrm{BD}$ ages are in disagreement with each other, and averaging the two values places the clusters in the intermediate $\log t=8.5$ age zone. Thus, the main features seen in the CMD-based age distribution are also seen in the age distribution determined from the integrated spectra.

We can examine the chemical evolution of the LMC by looking at the AMR for the star clusters. Given the short formation timescale for an individual cluster compared with the LMC lifetime, the metallicity of each cluster is a snapshot of the local chemical conditions at the time of formation. In Figure 25 we show the AMR for all clusters which have primary chemical abundance measurements from

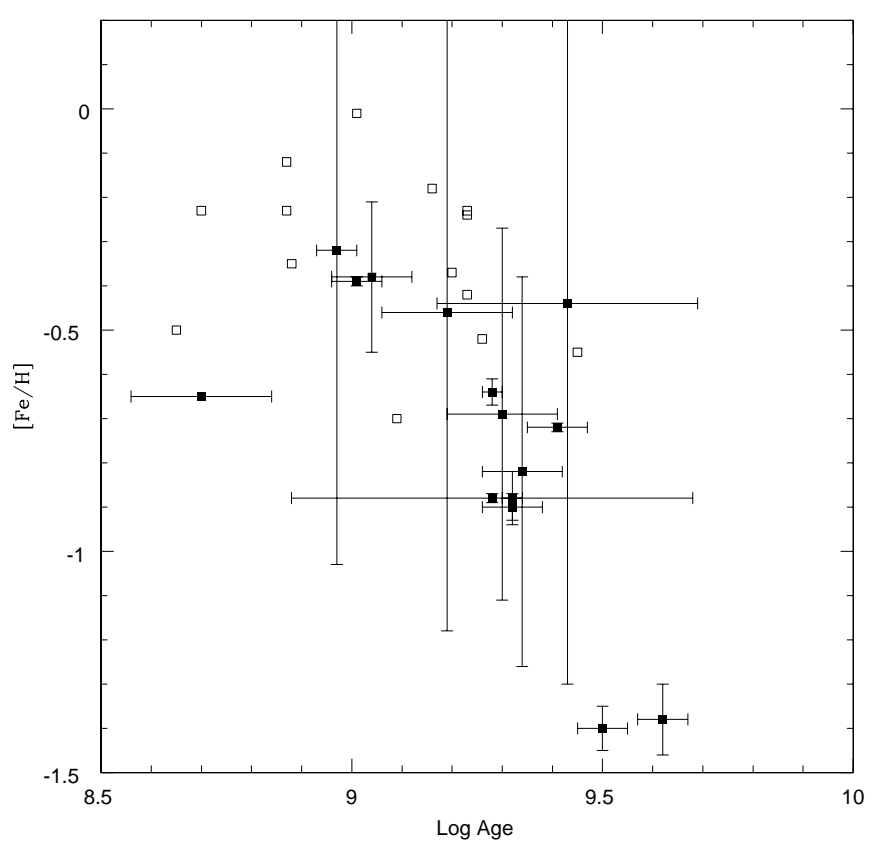

FIG. 25.-LMC age-metallicity relation for the derived parameters (filled squares) and the literature values (open squares) only using clusters with Olszewski et al. (1991) metallicity measurements.

Olszewski et al. (1991). The open squares represent the literature values (with $[\mathrm{Fe} / \mathrm{H}]$ coming from Olszewski et al. 1991 and the ages coming from CMD-based determinations), while the filled squares represent our determinations of age and $[\mathrm{Fe} / \mathrm{H}]$ for the same sample of clusters. The AMR derived from literature values is basically flat; i.e., no trend in $[\mathrm{Fe} / \mathrm{H}]$ with age and with considerable scatter in $[\mathrm{Fe} / \mathrm{H}]$ at all ages. The lack of a trend in the AMR is further supported by the recent Geisler et al. (2003) study. On the other hand, our determinations appear to support a significant AMR slope in the sense of steady enrichment over the past few gigayears from $[\mathrm{Fe} / \mathrm{H}]=-1.0$ up to $[\mathrm{Fe} /$ $\mathrm{H}]=-0.3$ at the present day. While such an enrichment pattern is perhaps not out of the question, we propose that it is likely to be an artifact of the correlated errors in age and metallicity that arise from errors in the observed spectral indices of the clusters. Moreover, as discussed in $\S 6.4$, in Figure 23 we find some indication that the discrepancy between our ages and the literature ages increases with decreasing metallicity, which has the effect of producing a spurious AMR. Clearly it would be interesting to obtain definitive $[\mathrm{Fe} / \mathrm{H}]$ values for the five clusters (NGC 1651, $1846,2162,2173$, and 2213) that occupy the older age, lower $[\mathrm{Fe} / \mathrm{H}]$ part of the plot for our determinations.

\section{CONCLUSIONS}

The principal result of this paper is that ages of young $(<4$ Gyr) star clusters can be determined through evolutionary synthesis modelling of the integrated light of the clusters. An effective discrimination of age from metallicity effects in most cases can be accomplished using a pair of spectral indices that leverage the different behavior of two Balmer lines $(\mathrm{H} \delta$ and $\mathrm{H} \epsilon$ ) against neighboring metal-line features. To adequately distinguish young ages in populations with $[\mathrm{Fe} / \mathrm{H}]<-1$, an index measuring the strength of the Balmer discontinuity needs to be incorporated as well. 
Age-metallicity degeneracy effects remain for young $(\leq 0.4$ Gyr $)$, metal-poor $([\mathrm{Fe} / \mathrm{H}]<-1)$ clusters. However, this degenerate region represents a relatively small portion of the entire age-metallicity parameter space. We base our conclusions regarding the reliability of age-dating young stellar populations from modelling of their integrated spectra on a comparison between synthetic spectral indices generated from evolutionary synthesis models and observed spectral indices in MC clusters. We find that the agreement between models and observations is particularly good in the case of the two line ratio indices, while the agreement is less convincing in the case of the Balmer discontinuity index. There is still uncertainty as to whether the disagreement is due to spectrophotometric errors or to deficiencies in the models. However, with the emphasis shifting to working as far into the rest-frame blue as possible, so as to perform such analyses at appreciable redshifts, the Balmer discontinuity index holds considerable promise, since observations of rest frame $3500 \AA$ are simplified at moderate redshift.

While this study has been largely successful at disentangling the effects of age and metallicity on the integrated spectra of young star clusters, the ultimate goal is to carry out such an analysis in the more general case, where a young poststarburst population is superposed on an underlying older population. Such an analysis has a variety of applications toward disentangling starburst scenarios in galaxies, which is becoming an increasingly important task for early epochs of galaxy evolution. In this case we are faced with the additional degeneracy of the ratio of burst light to underlying old light versus age of the burst. Specifically, a younger, weaker (relative to the old population) burst is difficult to distinguish from an older, stronger burst. Thus, in the more general case of a poststarburst population superposed on an older population there is the threefold degeneracy to consider of burst age, burst strength (relative to the old population), and chemical composition. The burst age versus burst strength degeneracy can be readily distinguished if one can assume solar composition for the population (LR96). Moreover, in the particular case of NGC 5018 the threefold degeneracy was effectively resolved (Leonardi $\&$ Worthey 2000), but largely as a result of the fact that, in this case, the starburst population completely dominated the integrated light within the spectrograph aperture. In short, additional work remains to be carried out if an entirely robust method of determining poststarburst ages is to be achieved.

We are greatly indebted to Dr. Robert Kurucz, for making available his stellar atmosphere models and SYNTHE code and to Dr. Guy Worthey for supplying his population synthesis code. We also thank the anonymous referee for careful reviews, which led to considerable improvements to the manuscript. This research was partially supported by National Science Foundation grants AST 93-20723 and AST 99-00720 to the University of North Carolina.

\section{REFERENCES}

Barbero, J., Brocato, E., Cassatella, A., Castellani, V., \& Geyer, E. H. 1990, ApJ, 351, 98

Beasley, M. A., Hoyle, F., \& Sharples, R. M. 2002, MNRAS, 336, 168

Bernlöhr, K. 1992, A\&A, 263, 54

Bertelli, G., Bressan, A., Chiosi, C., Fagotto, F., \& Nasi, E. 1994, A\&AS, 106,275

Bica, E., \& Alloin, D. 1986a, A\&A, 162, 21 1986b, A\&AS, 66, 171

Bica, E., Alloin, D., \& Santos, J. F. C., Jr. 1990, A\&A, 235, 103

Bica, E., Alloin, D., \& Schmidt, A. 1990, MNRAS, 242, 241

Bica, E., Geisler, D., Dottori, H., Claria, J. J., Piatti, A. E., \& Santos, J. F. C., Jr. 1998, AJ, 116, 723

Bica, E., Clariá, J. J., \& Dottori, H. 1992, AJ, 103, 1859

Bica, E., Clariá, J. J., Dottori, H., Santos, J. F. C., Jr., \& Piatti, A. E. 1996, ApJS, 102, 57

Bicker, J., Fritze-v. Alvensleben, U., \& Fricke, K. J. 2002, A\&A, 387, 412

Bruzual A., G. 1983, ApJ, 273, 105

Bruzual A., G., \& Charlot, S. 1993, ApJ, 405, 538

Butcher, H. R. 1977, ApJ, 216, 372

Butcher, H. R., \& Oemler, A., Jr. 1978a, ApJ, 219, 18

. 1978b, ApJ, 226, 559

Buzzoni, A., Mantegazza, L., \& Gariboldi, G. 1994, AJ, 107, 513

Charlot, S., \& Silk, J. 1994, ApJ, 432, 453

Charlot, S., Worthey, G., \& Bressan, A. J. 1996, ApJ, 457, 625

Cohen, J. G. 1982, ApJ, 258, 143

Cohen, J. G., Rich, R. M., \& Persson, S. E. 1984, ApJ, 285, 595

Couch, W. J., \& Sharples, R. M. 1987, MNRAS, 229, 423

Da Costa, G. S. 1991, in IAU Symp. 190, New Views of the Magellanic

Clouds, ed. Y.-H. Chu, J. E. Hesser, \& N. B. Suntzeff (Dordrecht:

Kluwer), 183

de Freitas Pacheco, J. A., Barbuy, B., \& Idiart, T. 1998, A\&A, 332, 19

Dirsch, B., Richtler, T., Gieren, W. P., \& Hilker, M. 2000, A\&A, 360, 133

Dressler, A., \& Gunn, J. E. 1983, ApJ, 270, 7

Durand, D., Hardy, E., \& Melnick, J. 1984, ApJ, 283, 552

Ellis, R. S. 1997, ARA\&A, 35, 389

Elson, R. A. W., \& Fall, S. M. 1985, ApJ, 299, 211 1988, AJ, 96, 1383

Faber, S. M., Friel, E. D., Burstein, D., \& Gaskell, C. M. 1985, ApJS, 57, 711

Feltzing, S., Holmberg, J., \& Hurley, J. R. 2001, A\&A, 377, 911

Frenk, C. S., \& Fall, S. M. 1982, MNRAS, 199, 565

Geha, M. C., et al. 1998, AJ, 115, 1045

Geisler, D., Bica, E., Dottori, H., Claria, J. J., Piatti, A. E., \& Santos, J. F. C., Jr. 1997, AJ, 114, 1920

Geisler, D., Piatti, A. E., Bica, E., \& Claria, J. J. 2003, MNRAS, 341, 771 Girardi, L., \& Bertelli, G. 1998, MNRAS, 300, 533

Girardi, L., Chiosi, C., Bertelli, G., \& Bressan, A. 1995, A\&A, 298, 87

Harris, J., \& Zaritsky, D. 2001, ApJS, 136, 25

Hill, V., Francois, P., Spite, M., Primas, F., \& Spite, F. 2000, A\&A, 364, L19

Hodge, P. W. 1981, in IAU Colloq. 68, Astrophysical Parameters for Globular Clusters, ed. A. G. D. Philip \& D. S. Hayes (Schenectady: L. Davis), 205

1982, ApJ, 256, 447

Holtzman, J. A. et al. 1999, AJ, 118, 2262

Jacobi, G. H., Hunter, D. A., \& Christian, C. A. 1984, ApJS, 56, 257

Jensen, J., Mould, J., \& Reid, N. 1988, ApJS, 67, 77

Jones, J. A. 1999, Ph.D. thesis, Univ. North Carolina Chapel Hill

Kennicutt, R. C., Jr., Roettgiger, K. A., Keel, W. A., van der Hulst, J. M., \& Hummel, E. 1987, AJ, 93, 1011

Kerber, L. O., Santiago, B. X., Castro, R., \& Valls-Gabaud, D. 2002, A\&A, 390, 121

Kontizas, M., Morgan, D. H., Hatzidimitriou, D., \& Kontizas, E. 1990, A\&AS, 84, 527

Kurth, O. M., Fritze-v. Alvensleben, U., \& Fricke, K. J. 1999, A\&AS, 138, 19

Kurucz, R. L. 1993, CD-ROM No. 18, SYNTHE Spectrum Synthesis Programs and Line Data (Cambridge: Smithsonian Astrophys. Obs.)

- 1994, Kurucz CD-ROM No. 19, Solar Abundances Model Atmospheres for 0, 1, 2, 4, $8 \mathrm{~km} / \mathrm{s}$ (Cambridge: Smithsonian Astrophys. Obs.)

Larson, R. B., \& Tinsley, B. M. 1978, ApJ, 219, 46

Leitherer, C., et al. 1996, PASP, 108, 996

Leonardi, A. J., \& Rose, J. A. 1996, AJ, 111, 182

Leonardi, A. J., \& Worthey, G. 2000, ApJ, 534, 650

Mclure, R. D. \& Tinsley, B. M. 1976, ApJ, 208, 480

Mihos, J. C., \& Hernquist, L. 1994, ApJ, 425, L13 1996, ApJ, 464, 641

Nolan, L. A., Dunlop, J. S., \& Jimenez, R. 2001, MNRAS, 323, 385

Olsen, K. A. G. 1999, AJ, 117, 2244

Olszewski, E. W., Harris, H. C., Schommer, R. A., \& Canterna, R. W. 1988, AJ, 95,84

Olszewski, E. W., Schommer, R. A., Suntzeff, N. B., \& Harris, H. C. 1991 , AJ, 101, 515

Piatti, A. E., Sarajedini, A., Geisler, D., Bica, E., \& Claria, J. J. 2002, MNRAS, 329, 556

Rabin, D. 1982, ApJ, 261, 85

Rich, R. M., Shara, M. M., \& Zurek, D. 2001, AJ, 122, 842 
Rifatto, A., Rafanelli, R., Ciroi, S., Radovich, M., Vennik, J., Richter, G., \& Birkle, K. 2001, AJ, 122, 2301

Rose, J. A. 1984, AJ, 89, 1238

1985, AJ, 90, 1927

1994, AJ, 107, 206

Rose, J. A., Stetson, P. B., \& Tripicco, M. J. 1987, AJ, 94, 1202

Sagar, R., \& Pandey, A. K. 1989, A\&AS, 79, 407

Schweizer, F., \& Seitzer, P. 1992, AJ, 104, 1039

Searle, L., Sargent, W. L. W., \& Bagnuolo, W. G. 1973, ApJ, 179, 427

Searle, L., Wilkinson, A., \& Bagnuolo, W. G. 1980, ApJ, 239, 803
Seggewiss, W., \& Richtler, T. 1989, in Recent Developments of Magellanic Cloud Research, ed. K. S. de Boer, F. Spite, \& G. Stasinska (Meudon: Obs. Paris) 45

Spite, M., Hill, V., Primas, F., Francois, P., \& Spite, F. 2001, NewA Rev., 45,557

Toomre, A., \& Toomre, J. 1972, ApJ, 178, 623

van den Bergh, S. 1991, ApJ, 369, 1

Welch, D. L. 1991, AJ, 101, 538

Worthey, G. 1994, ApJS, 95, 107

Worthey, G., \& Ottaviani, D. L. 1997, ApJS, 111, 377 